\title{
The Role of Entrainment in Human Walking: Energy Minimization in Oscillating Environments
}

Ryan Schroeder ( $\nabla$ ryan.schroeder@ucalgary.ca )

Biomedical Engineering, University of Calgary, Calgary, AB, Canada

James Croft

University of Calgary

John Bertram

University of Calgary

\section{Research Article}

Keywords: Entrainment, Coupled Oscillators, Human Locomotion, Energy Optimization

Posted Date: December 7th, 2020

DOl: https://doi.org/10.21203/rs.3.rs-120108/v1

License: (1) This work is licensed under a Creative Commons Attribution 4.0 International License.

Read Full License

Version of Record: A version of this preprint was published at Scientific Reports on August 4th, 2021. See the published version at https://doi.org/10.1038/s41598-021-95047-x. 
1 The Role of Entrainment in Human Walking: Energy Minimization in

2 Oscillating Environments

3 Ryan T. Schroeder ${ }^{1,2,3}$, James L. Croft ${ }^{3}$, and John E.A. Bertram ${ }^{1,2,4}$

4

$5 \quad{ }^{1}$ Biomedical Engineering, University of Calgary, Calgary, AB, Canada

$6 \quad{ }^{2}$ School of Medical and Health Sciences, Edith Cowan University, Perth, WA, Australia

$7 \quad{ }^{3}$ Faculty of Kinesiology, University of Calgary, Calgary, AB, Canada

$8{ }^{4}$ Cumming School of Medicine, University of Calgary, Calgary, AB, Canada

9

$10 *$ Ryan T. Schroeder

11 Email: ryan.schroeder@ucalgary.ca

12

13 R.T.S., 0000-0002-0613-3440; J.L.C., 0000-0002-7457-0837

14

15 Keywords

16 Entrainment, Coupled Oscillators, Human Locomotion, Energy Optimization

17

18

19

20 


\section{Abstract}

22 During locomotion, humans often entrain (i.e. synchronize) their steps to external oscillations:

23 e.g. swaying bridges, tandem walking, bouncy harnesses, vibrating treadmills, exoskeletons.

24 Previous studies have discussed the role of nonlinear oscillators (e.g. central pattern generators)

25 in facilitating entrainment. However, the underlying benefits of entrainment are unknown.

26 Given substantial evidence that humans prioritize economy during locomotion, we tested

27 whether reduced metabolic expenditure accompanies human entrainment to vertical force

28 oscillations, where frequency and amplitude were prescribed via a custom mechatronics system

29 during walking. Although metabolic cost was not significantly reduced during entrainment,

30 individuals who experienced negative work from oscillations had a higher cost than those who

31 experienced positive work, and subjects generally selected phase relationships indicating the

32 latter. It is possible that individuals use mechanical cues to infer energy cost and inform

33 effective gait strategies. If so, an accurate prediction may rely on the relative stability of

34 interactions with the environment. Our results suggest that entrainment is preferred over a

35 wide range of oscillation parameters, though not as a direct priority for minimizing metabolic

36 cost. Instead, entrainment may act to stabilize interactions with the environment, thus

37 increasing predictability for the effective implementation of internal models that guide energy

38 minimization. 


\section{Introduction}

47 Human walking is an oscillating system where the body moves in cyclic patterns to traverse a

48 substrate - often a static environment, e.g. a sidewalk. However, sometimes the environment

49 behaves as a second oscillating system. In this case, the human and the environment together

50 constitute a coupled oscillator system. For example, pedestrians sometimes spontaneously

51 synchronize the frequency of their steps with that of a swaying bridge as they $\operatorname{cross}^{1}$, and this

52 frequency matching is referred to as entrainment. A similar phenomenon occurs in laboratory

53 experiments where individuals are asked to walk on treadmills actuated with controlled

54 oscillations in mediolateral ${ }^{2}$ and vertical directions ${ }^{3,4}$. A recent study demonstrated human

55 entrainment with periodic electrical stimulations of the medial gastrocnemius while walking on

56 a conventional treadmill 5 . Furthermore, during development, infants can learn to entrain their

57 bouncing frequency with the resonance of an elastic harness ${ }^{6}$.

58 Ahn and Hogan ${ }^{7,8}$ interpreted subject entrainment with an ankle exoskeleton as evidence that

59 human locomotion is controlled, at least in part, by low level rhythmic nonlinear oscillators

60 (e.g. central pattern generators). In their study, an actuator provided periodic torque profiles at

61 the ankle joint independent of the subject's actions. Over time, subjects learned to entrain with

62 the periodicity of the exoskeleton and align muscle activation with that of the artificial system. It

63 is unclear whether such behaviors represent a simple quirk of control mechanisms driving

64 rhythmic gait, or if entrainment patterns are evidence of a more active process guiding effective

65 locomotion in dynamic environments.

66 A longstanding perspective on gait recognizes that preferred movement patterns are largely

67 consistent with energy minimization over a wide range of circumstances. There is strong

68 empirical evidence that individuals naturally select walking parameters (step frequency, step

69 length, step width, etc.) that minimize metabolic energy per distance travelled ${ }^{9-12}$. Furthermore,

70 it appears that internal models are continuously updated in real time to optimize energy output

71 under novel circumstances ${ }^{13-15}$.

72 Here, we test if entrainment to external oscillations is motivated by a reduction in metabolic

73 expenditure. To accomplish this, we used a mechatronics oscillator system to provide periodic

74 vertical forces (upward and downward) to the trunks of subjects as they walked on a treadmill

75 (Fig. 1). As opposed to an exoskeleton strapped to a single joint, the oscillation system in this 
study is used to more directly influence the center of mass (CoM) and its dynamics - arguably a

77 fundamental aspect of the task of locomotion ${ }^{16}$ - to directly assess locomotor control strategies driving interactions within a dynamic environment.

79 The system used two linear servomotors to tug on a pulley-cable system connected to a body

80 harness worn by subjects (Fig. 1). An open loop current control was prescribed to the motors

81 during experiments where the oscillation frequency and amplitude were fixed at different values

82 for each trial condition. A more detailed description of the system can be found in the

83 Supplementary Materials.

a.

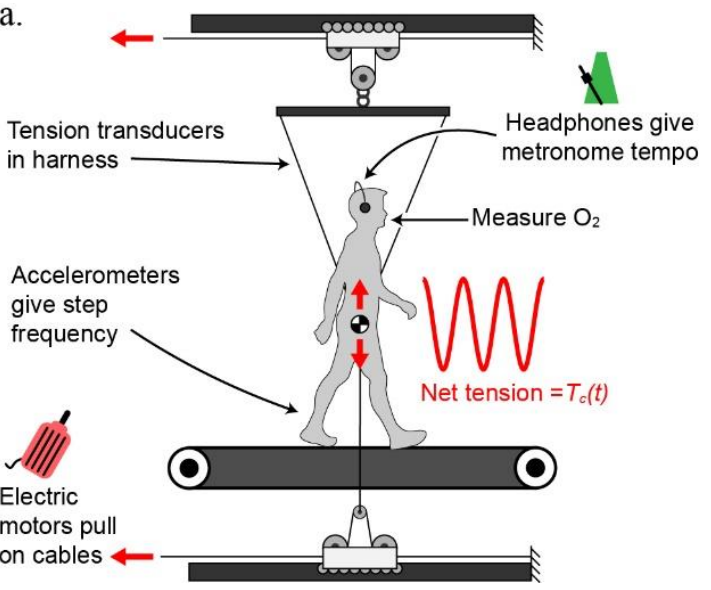

b.

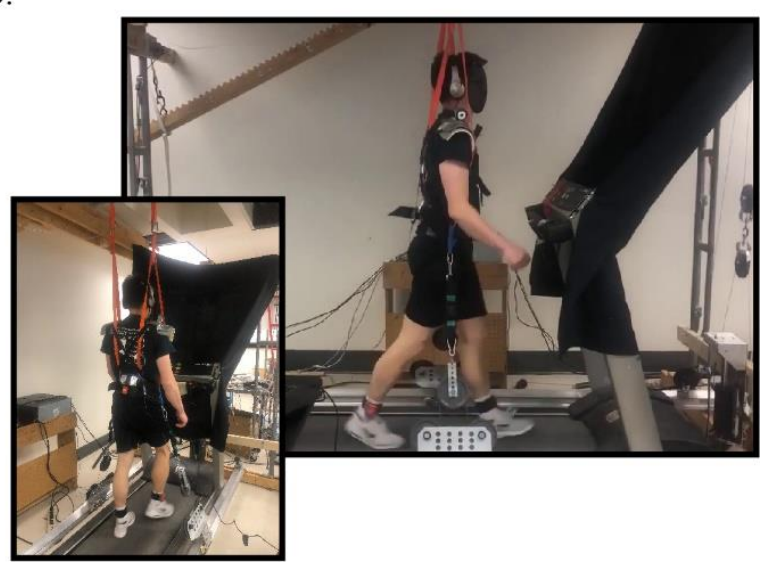

Figure 1. System schematic and images. (a) A schematic of the oscillator system is depicted in the sagittal plane. (b) Images of a subject walking in the system during a trial, from the side and from behind. Downward force came as the resultant of self-equalizing oblique cables. A curtain was used to blind the subject from any motion of the pulleys or motors, and headphones were used to play ambient noise to block out rhythmic sounds from the system. The headphones were also used to play a metronome beep during portions of the experiment. A more detailed description of the system can be found in the Supplementary Materials.

The experiment was divided into two phases while external oscillations were present (Fig. 2): first, subjects walked with a freely-chosen step frequency in response to the oscillations (i.e. individuals were allowed to entrain to the oscillations); second, individuals followed the beat of a metronome programmed to subject-specific baseline frequencies, measured a priori. Both experiment phases lasted five minutes and allowed for a comparison of energetic 
99 force oscillations with the gait cycle were measured to characterize subject-preferred mechanical interactions with the oscillating environment.

a.

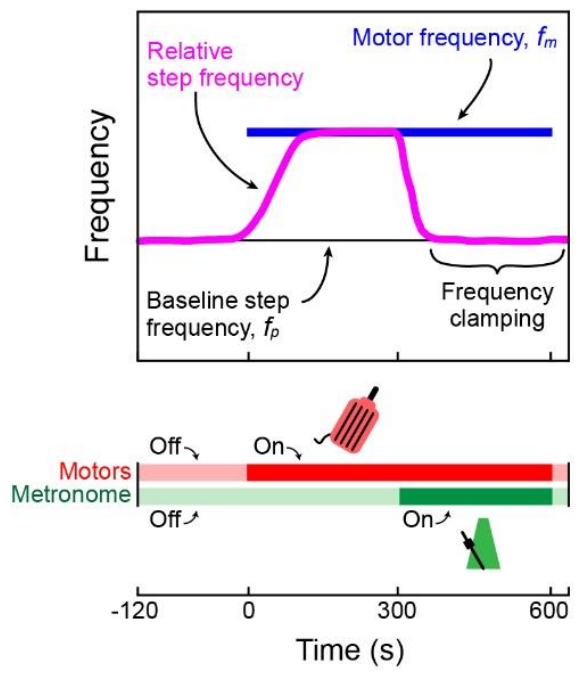

b.
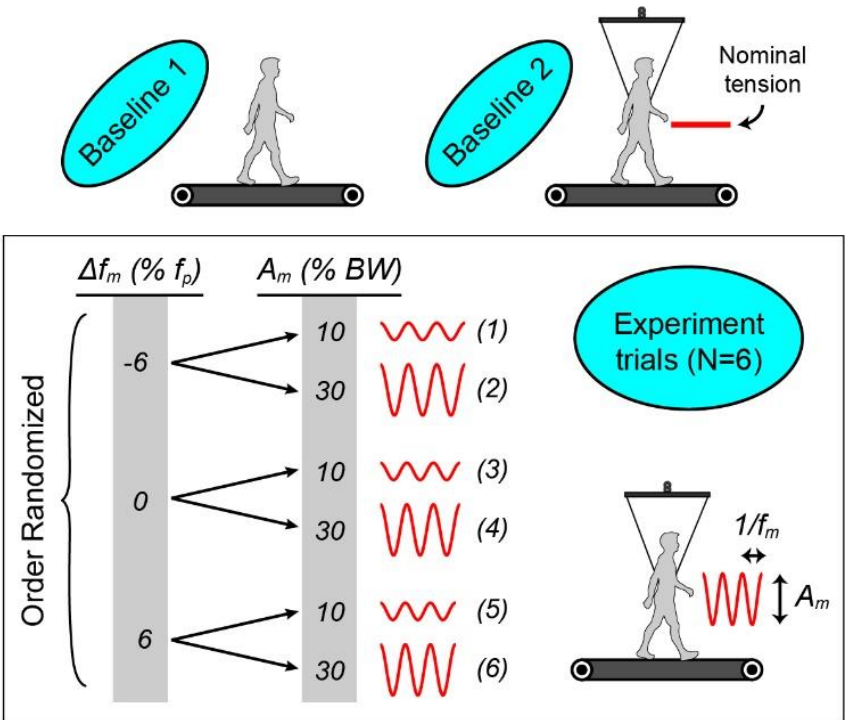

102

103

104

105

106

Figure 2. Experimental protocol. (a) A generic trial condition is depicted with simulated step frequency data over time (magenta) and constant motor frequency (blue). Subjects walk with no oscillations during the first two minutes of the test. Motor oscillations begin at time zero and continue for five minutes while subjects freely interact with the system. Oscillations continue for another five minutes, but now a metronome directs individuals to step at their baseline preferred frequency $\left(f_{p}\right)$ despite the external oscillation frequency ("frequency clamping"). The oscillations and metronome are terminated, and the subject is given fifteen additional seconds to prepare for the end of the trial. (b) Baseline conditions and oscillation parameters during experiment trials are shown: $\Delta f_{m}=0, \pm 6 \%$ and $A_{m}=10,30 \%$ body weight (BW). Trial conditions were implemented randomly to reduce ordering effects. 


\section{Results}

\section{Subjects entrain to external oscillations}

116 Figure 3 shows the median step frequency normalized to baseline preferred (magenta) as well

117 as $25 \%$ and $75 \%$ quartiles (grey shaded region) for individuals who entrained their steps with

118 external oscillations at least once during the indicated trial condition. Subjects generally

119 preferred entrainment, overall. However, the level of entrainment varied between individuals;

120 e.g. some only entrained in two trials while others entrained in five out of six total trials. The

121 likelihood of subject entrainment largely depended on the oscillation parameters prescribed:

122 frequency, $\Delta f_{m}$, expressed as a percent difference from subject baseline and amplitude; $A_{m}$,

123 expressed as a percentage of subject body weight (BW) force (see Methods for details). For

124 example, all ten subjects entrained when $\Delta f_{m}=-6,0 \%$ and $A_{m}=30 \% B W$ (Fig. 3b,d).

125 Conversely, no subjects entrained when $\Delta f_{m}=6 \%$ and $A_{m}=10 \% B W$, thus only individual

126 subject data are shown (Fig. 3e). In general, entrainment in conditions with higher motor

127 frequencies and lower amplitudes was less stable and more transient. Note, the data for $\Delta f_{m}=$

1280 end earlier than other trials since there was no metronome used (the oscillation frequency

129 already matched baseline preferred, so was deemed unnecessary; Fig. 3c,d). However,

130 individuals largely followed the metronome in other trials, as the median data quickly converged

131 on a relative frequency of one at approximately $300 \mathrm{~s}$ into the trial. 

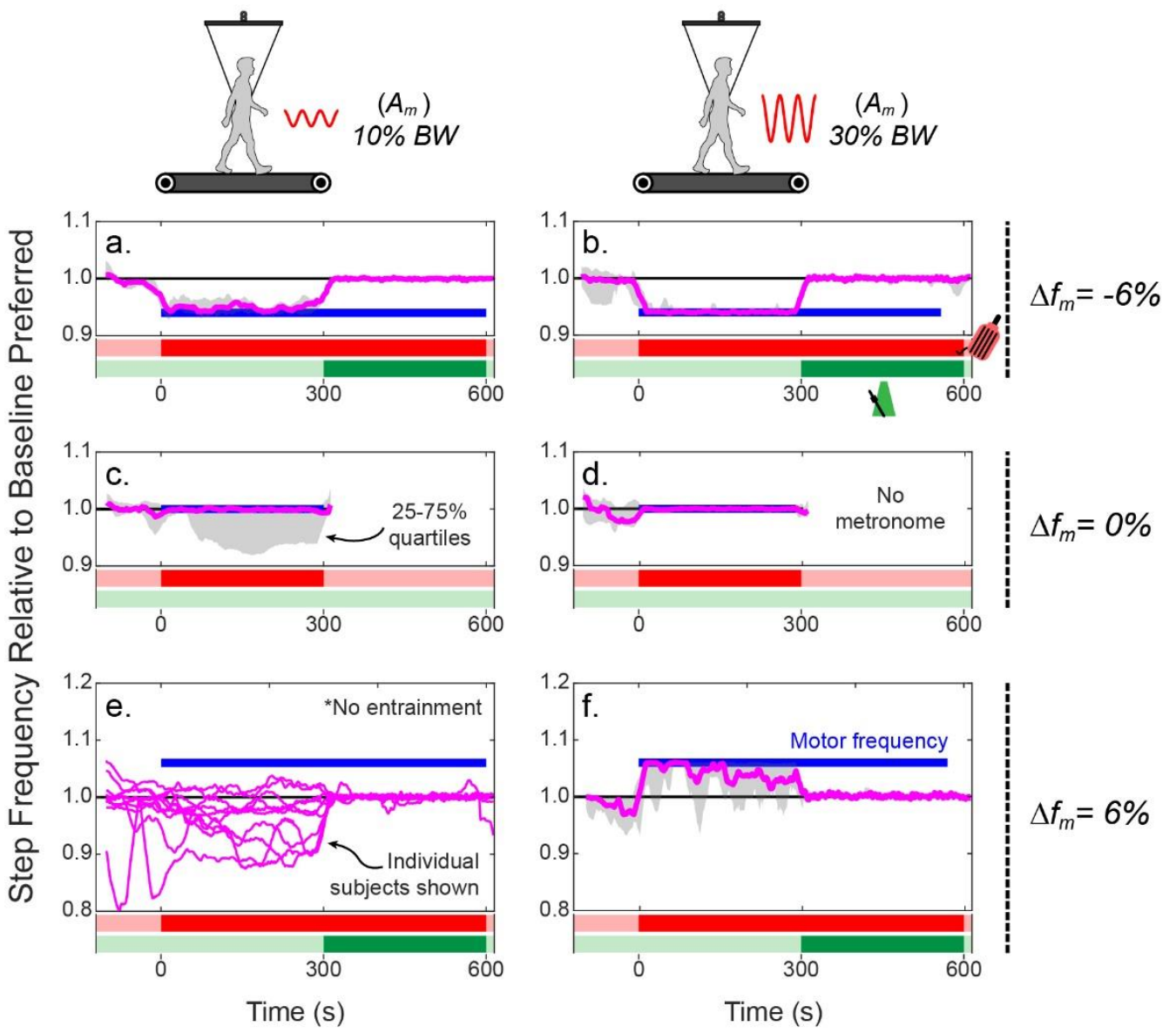

Figure 3. Entrainment results. The median relative step frequency $\left(f_{r}\right.$, step frequency divided by

134 preferred step frequency in Baseline 2; magenta) of all subjects who entrained is plotted over 135 each trial duration. $25 \%$ and $75 \%$ quartiles are used to indicate the distribution at every time 136 point (grey shaded area). All trial conditions are shown, including: $\Delta f_{m}=-6 \%$ in (a) and (b); $137 \Delta f_{m}=0 \%$ in (c) and (d); $\Delta f_{m}=6 \%$ in (e) and (f); $A_{m}=10 \% B W$ in (a), (c) and (e); $A_{m}=$ $13830 \% B W$ in (b), (d) and (f). The oscillations began at Time $=0 \mathrm{~s}$ and ended at approximately 139 Time $=600 \mathrm{~s}$. During $0 \leq$ Time $\leq 300 \mathrm{~s}$, subjects responded freely to the force oscillations. 140 During $300 \leq$ Time $\leq 600 \mathrm{~s}$, subjects were directed to follow the cadence of the metronome 141 at their predetermined baseline step frequency ("frequency clamping") even as the oscillations 142 continued at a different frequency. There was no metronome used in trials where $\Delta f_{m}=0$, 143 since frequencies were already matched. As a result, these experiments ended after around 144 Time $=300 \mathrm{~s}$. Note, median data are only shown for individuals who entrained at least once 145 throughout the trial. In the trial condition where $\Delta f_{m}=6 \%$ and $A_{m}=10 \% B W$, individual 146 subject data are shown instead since no entrainment occurred. 
In many instances, subjects exhibited transient entrainment - meaning their step frequency drifted in and out of the oscillation frequency throughout the trial (Fig. 4a). To better characterize how well subjects entrained their gait in the various trial conditions, two metrics were considered: entrainment step ratio ( $E S R, \mathrm{Fig} .4 \mathrm{~b})$ and average entrainment duration $\left(\Delta \bar{t}_{e}\right.$,

151 Fig. 4c). The entrainment ratio is the proportion of steps within \pm 3 standard deviations (SD) of 152 the motor frequency (1 SD determined from step frequency data in Baseline 2) during the first 153 five minutes of exposure to oscillations in the experiment (i.e. no metronome). However, 154 because this metric does not consider how bouts of transient entrainment are distributed 155 throughout the trial, $\Delta \bar{t}_{e}$ indicates the average time duration of all bouts in a given trial as a 156 fraction of the 300 seconds time allotted. Thus, for both entrainment metrics, a value of zero means that no entrainment occurred while a value of one means that subjects entrained throughout the entire trial.

a.

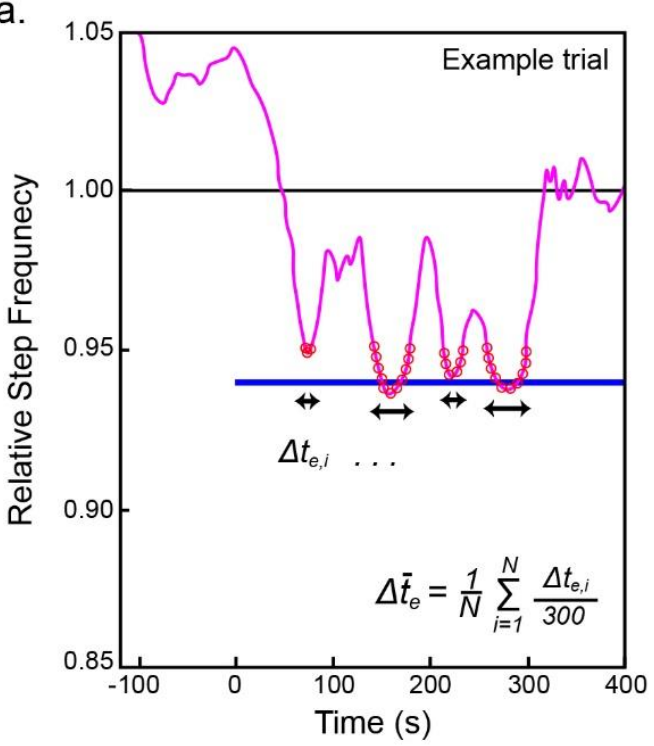

b.

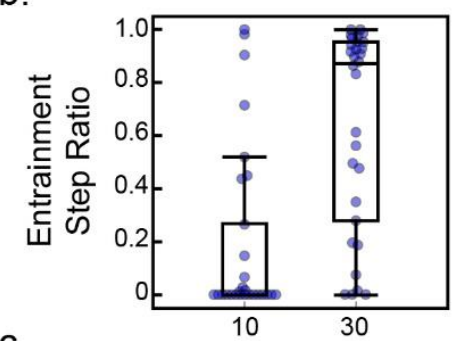

c.

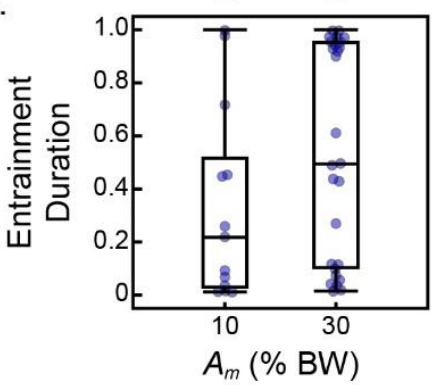

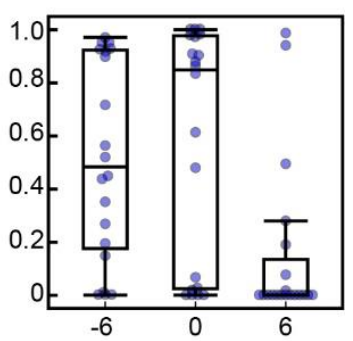

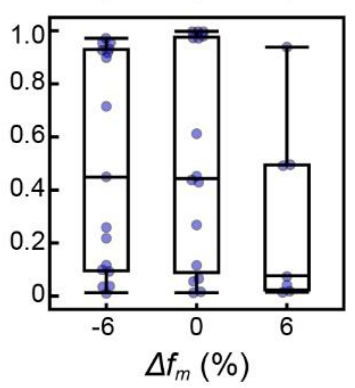

Figure 4. Entrainment is often transient. (a) Data from an example subject illustrates transient entrainment where relative step frequency (magenta) oscillates towards and away from the motor frequency (blue). Red data points indicate when the subject is considered entrained with

163 the oscillator system (see Methods section for details on entrainment definition). (b) The 164 entrainment step ratio ( $E S R$; ratio of entrained steps to total steps taken during the first five 165 minutes of oscillations in the experiment) and (c) the average entrainment duration $\left(\Delta \bar{t}_{e}\right.$; 166 average time duration of bouts of entrainment) are shown as a function of oscillation amplitude 167 and motor frequency, where each data point represents a subject's level of entrainment during 
each trial and box plots summarize the distribution. Linear mixed models were used to statistically test the effects of trial conditions on both entrainment metrics shown here (see Table S1 in the Supplementary Materials for full results).

Linear mixed models indicated that higher oscillation amplitudes increased both ESR [fitted coefficient (95\% confidence limits): $\beta=2.267$ (1.482, 3.052), $\left.p<0.001^{*}\right]$ and $\Delta \bar{t}_{e}[\beta=$ $\left.1.737(0.958,2.517), p<0.001^{*}\right]$ while higher motor frequencies led to decreases in the entrainment metrics: $\left[\beta=-3.005(-4.607,-1.402), p<0.001^{*}\right]$ and $[\beta=$ $-2.712(-4.302,-1.121), p=0.001^{*}$ ], respectively. An interaction between motor frequency and oscillation amplitude was not significant $(p=0.273)$. In fact, this interaction was not significant in any of the models tested. Figures $4 \mathrm{~b}$ and $4 \mathrm{c}$ illustrate higher levels of entrainment at larger amplitudes and lower motor frequencies, despite large inter-subject variation overall.

\section{Entrainment does not reduce metabolic power}

Metabolic power was compared for individuals in all trial conditions with the metronome turned off (subject allowed to entrain) versus with the metronome turned on (not allowed to entrain). Metabolic expenditure increased by $25.8 \%(p<0.001 *)$ when subjects walked on the treadmill wearing the harness but with no active oscillations versus when they walked on the treadmill without the harness (Baselines 2 and 1, respectively; Fig. 5). When comparing trials with active oscillations, no significant differences were found, with the exception of one parameter combination: $\Delta f_{m}=-6 \%$ and $A_{m}=30 \% B W$. This condition was more costly without the metronome compared to all other trials and baseline conditions. Still, metabolic cost did not differ significantly depending on the presence of the metronome (blue vs. green in Fig. 5) for any of the trial conditions tested. All in all, the metronome - and thus, the freedom of subjects to entrain - had no statistical effect on metabolic power. Importantly, this result did not change when controlling for the level of entrainment (e.g. ESR) in each trial condition. 


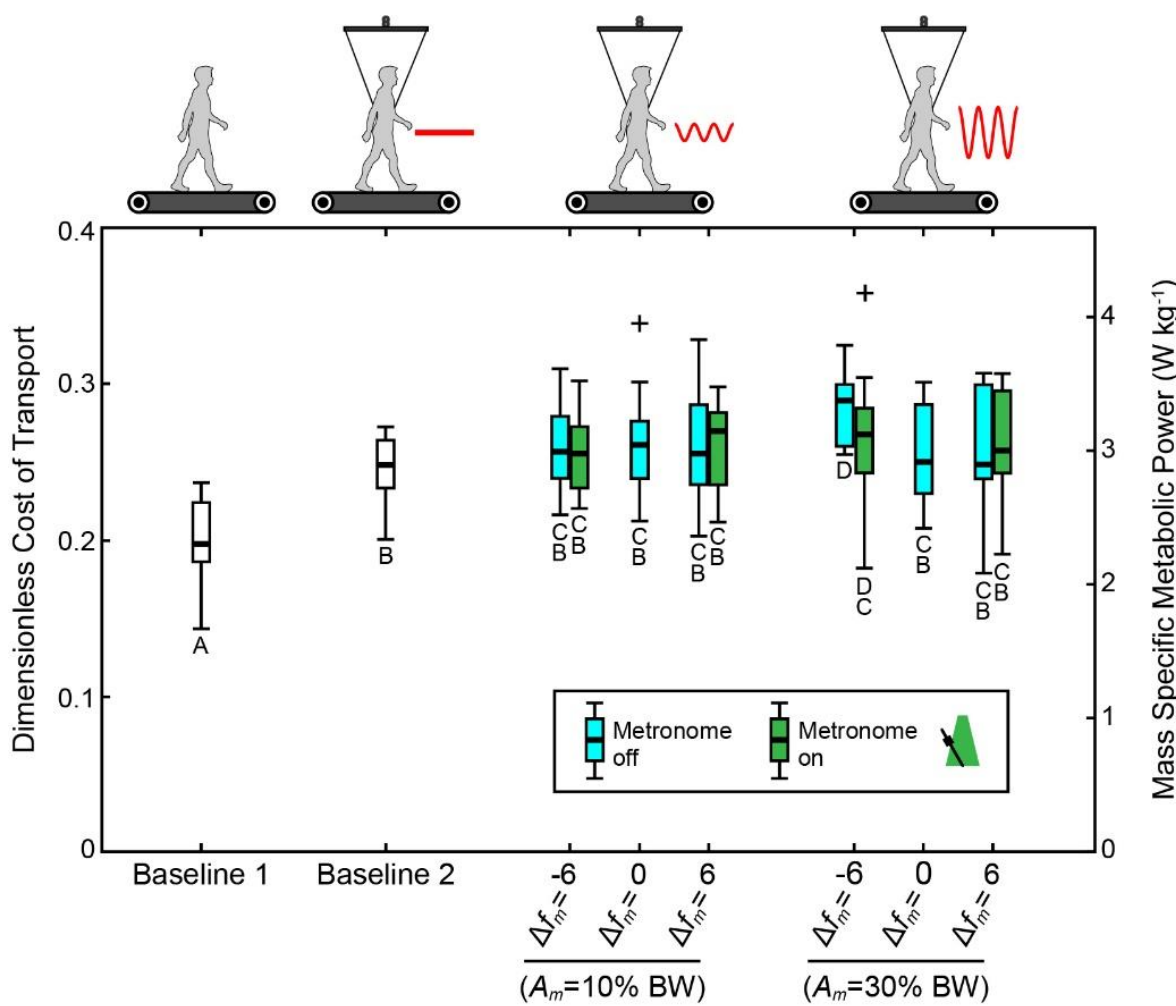

196 Figure 5. Metabolic power does not depend on entrainment. Non-dimensional metabolic power

197 was compared using a mixed linear regression stratified by trial condition and baseline type. A

198 post hoc Tukey Honestly Significant Difference test was used to compare estimates of metabolic 199 power in the model. Box plots are labelled with letters indicating conditions where power is not 200 significantly different. Outliers are marked with "+". See Table S2 in the Supplementary

201 Materials for full results. Baseline 1 and 2 refer to walking on the treadmill without and with the 202 harness, respectively. Box plots are shown for all metabolic data collected during the first five 203 minutes of oscillations where the subject freely responded to the oscillation forces (i.e.

204 metronome off). During the next five minutes of oscillations, a metronome guided subjects to 205 step at their baseline preferred frequency (importantly, not matched to the oscillation 206 frequency). This allowed for metabolic cost comparisons between entrained and non-entrained 207 gait. 


\section{Subjects prefer to align peak forces at toe off}

210 Figure 6a shows average tension forces measured in the harness for all subjects during

211 Baseline 2. These forces act to pull vertically near the CoM of subjects as they walk on the

212 treadmill. The red and blue curves indicate tension in the harness pulling up $\left(T_{\uparrow}\right)$ and down $\left(T_{\downarrow}\right)$,

213 respectively, while the black curve is the summation of the two $\left(T_{n e t}\right)$. Even though no active

214 oscillations occur during the baseline test, there are still fluctuations in tension due to passive

215 resistance of the system; these resistive forces largely occur due to the reflected inertia of the

216 motors and associated hardware, as well as to damping effects. Given the alignment of these

217 forces with the vertical velocity of the CoM (Fig. 6c), net negative power dominates the

218 interaction (black shaded area in Fig. 6 e, $\left.P_{\text {net }}\right)$ even as power of the cables pulling up $\left(P_{\uparrow}\right.$, red)

219 and down $\left(P_{\downarrow}\right.$, blue) partly offset each other with both positive and negative power.

220 In Figure 6b, average tension forces are shown for subjects entrained with active oscillations in

221 the system. Although the passive force peaks observed in Baseline 2 are still present in the

222 entrained interaction (occurs in $T_{\uparrow}$ at around 0.80 of the step cycle), there is an additional

223 upward peak ( 0.35 in the step cycle) due to subjects' preferred alignment of the active

224 oscillations. This active force peak is further indicated by the motor phase selected by subjects

225 during entrainment. The red histogram in Figure $6 \mathrm{~d}$ characterizes the distribution of motor

226 phase chosen by subjects during entrainment, where the average and standard deviation are

227 given: $\bar{\phi}=94 \pm 55^{\circ}$. This means that peak current is prescribed to the motor pulling up just

228 after a quarter through the step cycle (approximately at toe off of the trailing leg). Due to

229 system dynamics, this current peak shows up as a spike in tension force slightly later as the

230 "active force peak".

231 The preferred motor phase seems to imply a strategy of receiving positive power from the 232 active oscillations, given peak active force approximately aligns with peak vertical CoM velocity 233 (Fig. 6d). Despite this alignment, negative power still greatly outweighs any positive power 234 received from the system. Since the resistive forces largely responsible for excess negative 235 power relate to motion of the CoM (damping relates to velocity, inertial forces to acceleration), 236 a 90\% increase in vertical velocity amplitude may help to explain why net negative power still 237 dominates subjects during entrainment, despite a preferred phase indicating the opposite. 

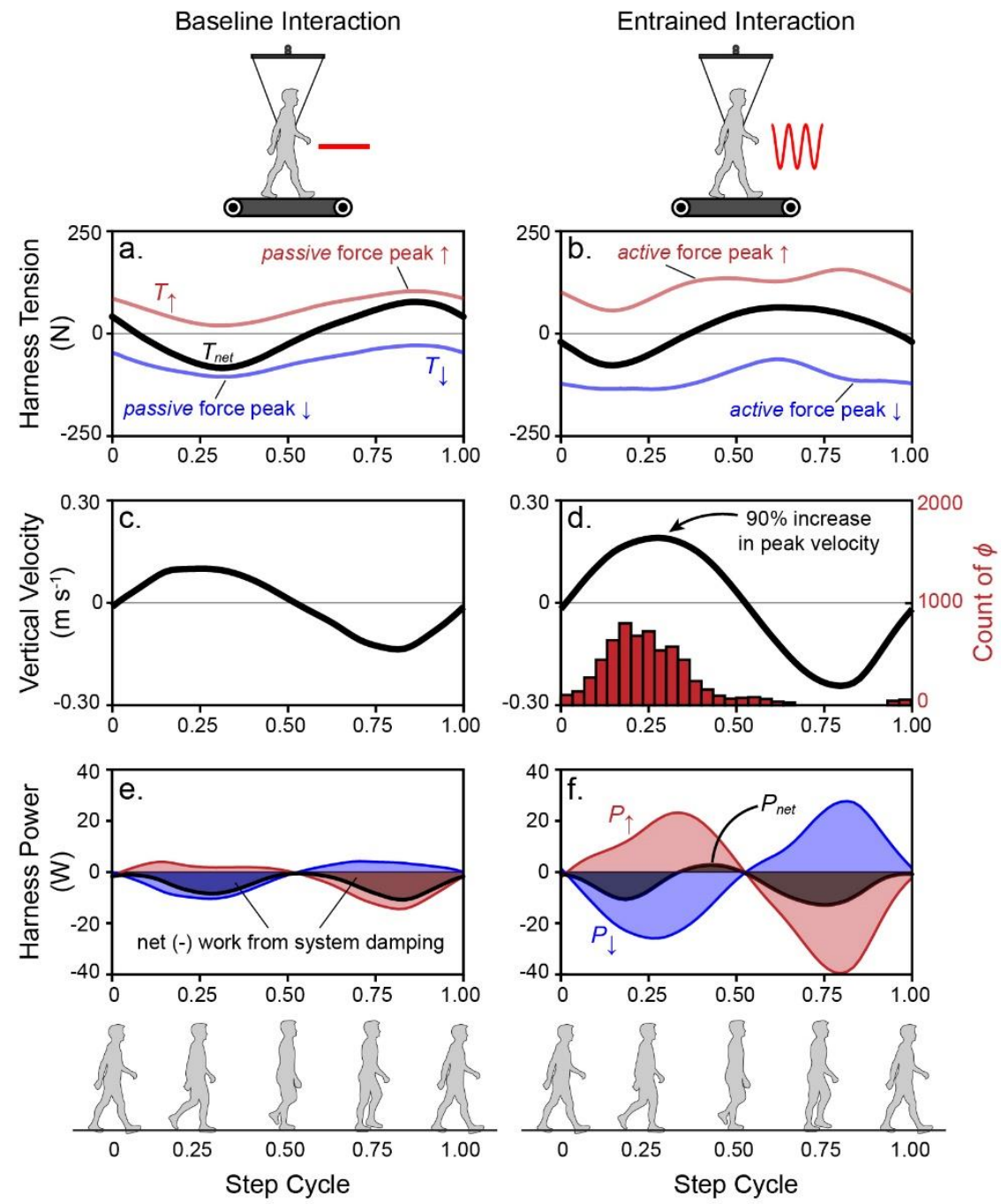

Figure 6. Subjects prefer to align peak oscillation forces at toe off. The average harness tension

241 is shown in (a) and (b), where the red and blue curves indicate cable tension pulling up $\left(T_{\uparrow}\right)$ and

242 down $\left(T_{\downarrow}\right)$, respectively, and black indicates net tension $\left(T_{\text {net }}\right)$. Average center of mass vertical

243 velocity is shown in (c) and (d), while the red histogram in (d) indicates the distribution of motor

244 phase chosen by subjects during entrainment. Average power from the cables pulling up $\left(P_{\uparrow}\right.$,

245 red) and down $\left(P_{\downarrow}\right.$, blue) are shown in (e) and (f), while net power $\left(P_{\text {net }}\right)$ is indicated with black

246 shading. Panels (a), (c) and (e) indicate average curves measured during Baseline 2 (walking with

247 the harness but no active oscillations), while panels (b), (d) and (f) indicate average curves

248 measured during experiment trials. Snapshots of the walking step cycle are shown near the

249 bottom to help orient the reader to the timing of events shown in plots. Zero in the step cycle

250 corresponds with double stance while 0.5 corresponds with single stance. 


\section{Preferred phase lag varies by subject}

252 Despite the entrainment strategy shown in Figure 6 representing the sample mean tested in the 253 experiment, individual subjects displayed notable variance - for example, with respect to the 254 work done by the oscillation forces on the CoM. Figure 7 shows the average tension force, CoM 255 vertical velocity and mechanical power of three example subjects in trial conditions chosen to

256 illustrate entrainment strategies resulting in moderate net positive work (Subject A), moderate 257 net negative work (Subject B) and substantial net negative work (Subject C). A notable 258 distinction of these subjects is the chosen phase alignment of motor forces. Subject $A$ aligned 259 the motor phase just after zero (median phase $=31^{\circ}$, or 0.087 in the gait cycle), and distinctive 260 tension force humps were observed shortly thereafter ( 0.25 in the gait cycle). Due to this 261 phase, the net tension signal was locally shaped by active oscillation forces and approximately 262 aligned with CoM velocity, thus resulting in net positive work $\left(W_{c}=4.1 \mathrm{~J}\right)$. Subject B preferred a 263 slightly increased phase $\left(64^{\circ}\right.$, or 0.18$)$. Although the resulting motor force hump was still 264 relatively distinct, it was not robust enough to overcome resistive forces due to inertia and 265 damping in the system, and net negative work was accumulated over the step $\left(W_{c}=-6.2 \mathrm{~J}\right)$. 266 Subject $C$ preferred a relatively late motor phase $\left(110.0^{\circ}\right.$, or 0.32$)$, and forces out of phase with 267 the CoM velocity were exaggerated. Substantial net negative work was observed in this example $268\left(W_{c}=-12 \mathrm{~J}\right)$. Data from example subjects in Figure 7 imply that motor phase alignment had a 269 prominent effect on the net mechanical work done on the CoM by the harness tension forces. 

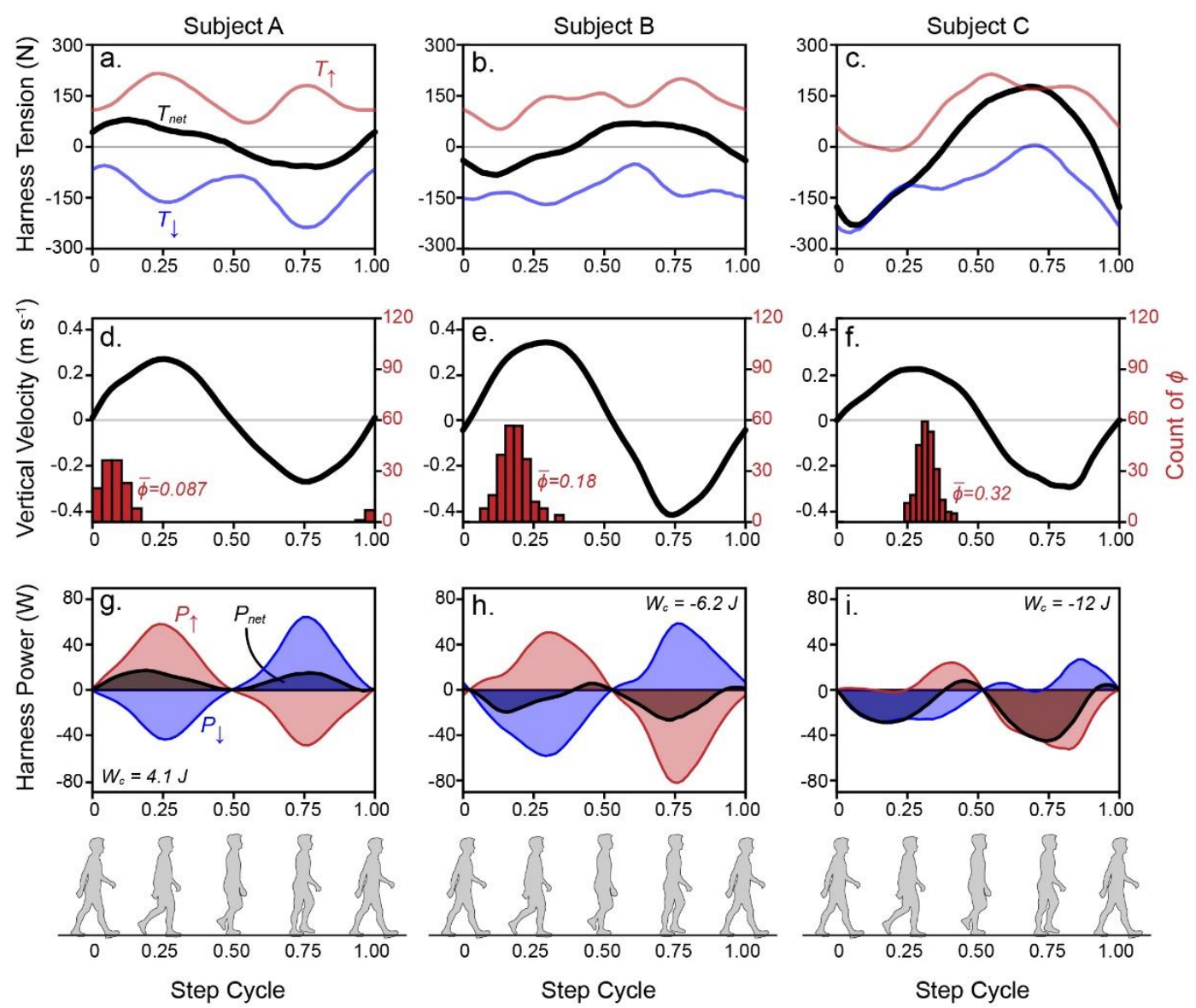

271 Figure 7. Subjects prefer a range of phase alignments, resulting in variable net power. Data

272 from three subjects were chosen to demonstrate varying entrainment strategies. Average data

273 for Subject A ( $\Delta f_{m}=6 \%, A_{m}=30 \% B W, 297$ steps averaged) are shown in (a), (d) and (g),

274 where motor phase alignment occurs at $\bar{\phi}=0.087$ of the gait cycle. This strategy aligns peak

275 tension forces with the center of mass (CoM) vertical velocity and thus, results in net positive

276 work $\left(W_{c}=4.1 \mathrm{~J}\right)$. Average data for Subject B $\left(\Delta f_{m}=-6 \%, A_{m}=30 \% B W, 475\right.$ steps

277 averaged) are shown in (b), (e) and (h) where motor phase alignment occurs at $\bar{\phi}=0.18$ of the

278 gait cycle. Since peaks in tension due to motor forces occur slightly later, positive power is

279 relatively low and net negative work occurs $\left(W_{c}=-6.2 \mathrm{~J}\right)$. Average data for Subject $\mathrm{C}\left(\Delta f_{m}=\right.$

$280-6 \%, A_{m}=30 \% B W, 444$ steps averaged) are in (c), (f) and (i) where $\bar{\phi}=0.32$ of the gait

281 cycle, and mechanical power from the harness tension is dominated by resistive inertial forces,

282 thus leading to substantial net negative work on the $\operatorname{CoM}\left(W_{c}=-12 \mathrm{~J}\right)$. Data points for subjects

283 A, B and C are labelled in Figure 8b. 


\section{Net mechanical work determines metabolic power}

286 Figure 8a shows how metabolic power and net mechanical work of the oscillations on the CoM

287 vary due to phase lag of the active peak force pulling up. Minimum metabolic power is observed

288 when active oscillations pull up on the body during toe off of the trailing leg $\left(\phi \approx 90^{\circ}\right)$, and this

289 corresponds to maximum net positive work from the oscillator forces (Fig. 8a). Although

290 subjects interacting with the oscillator system mostly experienced net negative mechanical work

291 overall, variation on the gait strategy chosen illustrated a range of work done

292 [approximately $-0.16-0.05 \mathrm{~W} \mathrm{~kg}^{-1}$ ] and a linear mixed model found a strong negative effect

293 of net work on metabolic cost in individual subjects and trial conditions $[\beta=$

$294-4.201(-4.960,-3.442), p<0.001 *$; see Fig. 8b]. In other words, more net positive work done

295 by the harness tension meant a lower metabolic cost for subjects and more net negative work

296 meant a higher cost. The oscillation amplitude also had an effect on metabolic power [ $\beta=$

$\left.2970.088(0.051,0.125), p<0.001^{*}\right]$, showing an increase in cost at higher amplitudes, despite

298 there being generally more entrainment in this condition. In fact, the level of entrainment was

299 controlled for by including ESR as a covariate in the model, and yet, this variable did not have a

300 significant effect on metabolic power $[\beta=-0.004(-0.013,0.006), p=0.488]$. An interaction

301 term between motor frequency and oscillation amplitude was found to be insignificant after

302 controlling for multiple hypothesis testing (see Table S1 in the Supplementary Materials for full

303 results). 

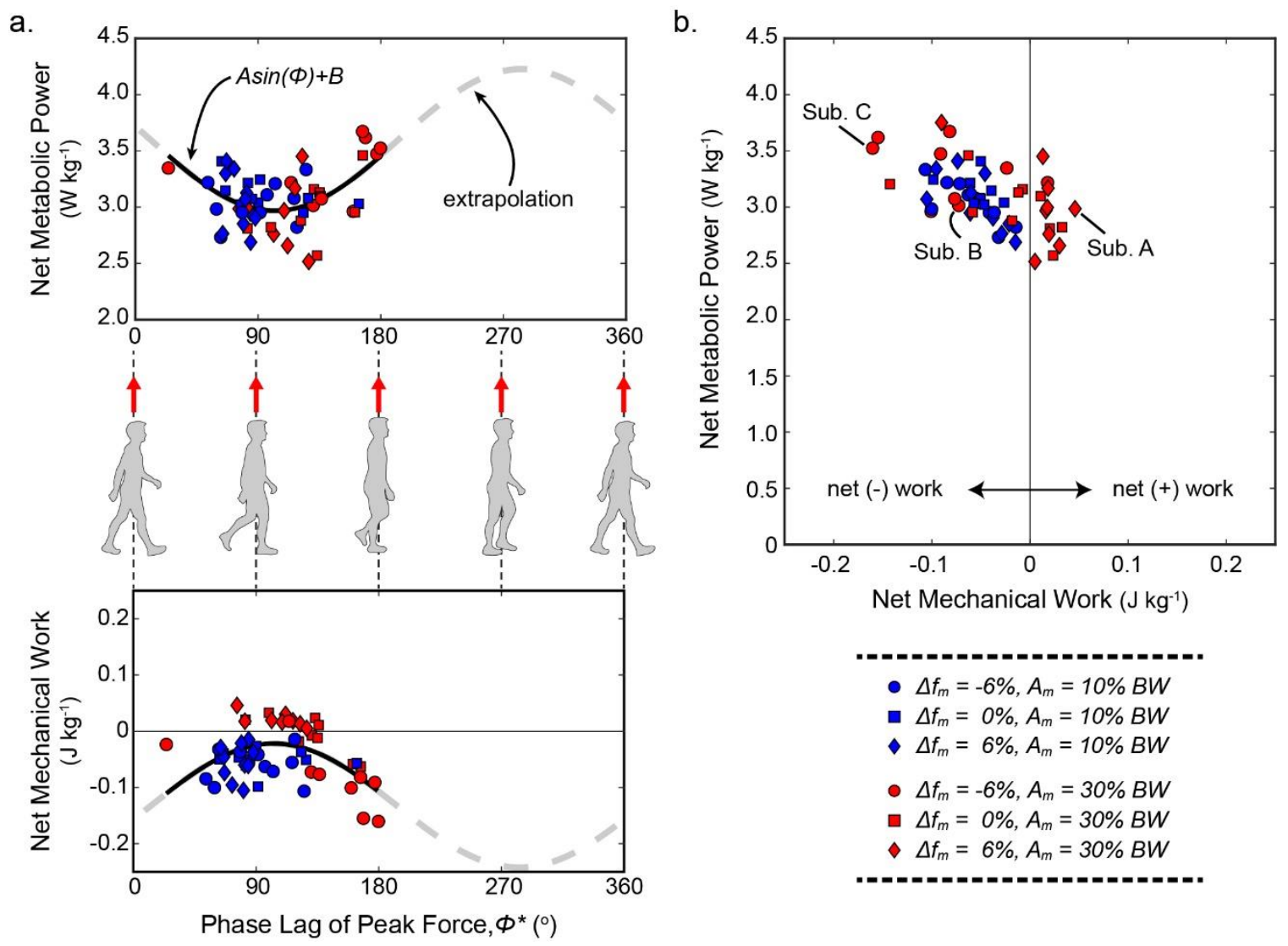

Figure 8. Positive mechanical work from the oscillator decreases metabolic power. Average data are shown for every subject in each trial condition during the first five minutes of oscillations when no metronome is present. (a) Net metabolic power is plotted versus the phase lag of peak upward force from the oscillations. A phase lag of $0^{\circ}$ (or $\left.360^{\circ}\right)$ corresponds to peak upward force occurring at double stance while a lag of $180^{\circ}$ corresponds to peak upward force

311 occurring at midstance (i.e. when the center of mass passes over the stance foot). Net

312 mechanical work done on subjects by the oscillation forces is also plotted versus the phase lag

313 of peak upward force. Arbitrary sinusoidal functions are fit to these data to indicate the cyclical

314 relationship between center of mass motion and oscillation forces. (b) Net metabolic power

315 decreases as a function of net mechanical work done on subjects by the oscillation forces. A

316 linear mixed model was used to assess the effect of net work on metabolic power (both

317 variables non-dimensionalized; see results in Table S1 in the Supplementary Materials). Example

318 data points from subjects A, B and C are labelled for comparison in plots of Figure 7. Blue and

319 red data points correspond to trial conditions where $A_{m}=10,30 \% B W$ respectively. Circles,

320 squares and diamonds correspond to trial conditions where $\Delta f_{m}=-6,0,6 \%$ respectively. 


\section{Discussion}

\section{Step frequency adaptations}

323 Step frequency adaptations in the current study ( $\pm 6 \%)$ are comparable to those of previous

324 reports: approximately $\pm 2-8 \%$ of preferred step frequency ${ }^{13-15,17}$. Even so, some subjects

325 struggled to entrain with the oscillator system even in the most favorable conditions (e.g.

326 preferred frequency, high amplitude). Similar variability in subject response has been noted in

327 other studies ${ }^{14}$. Regardless, clear trends were identified; subjects displayed the most robust and

328 stable entrainment during trials with high motor amplitudes and frequencies below the

329 preferred step frequency measured at baseline.

\section{Stronger entrainment at lower frequencies}

331 In general, subjects had an easier time entraining to oscillation frequencies below their

332 preferred step frequency. The entrainment step ratio and the average entrainment duration

333 were both found to be negatively affected by motor frequency ( $\beta=-3.005$ and -2.712 ,

334 respectively) - meaning lower frequency conditions were associated with more consistent and

335 robust entrainment and higher frequency conditions were associated with less consistent and

336 robust entrainment. It is unclear why stronger entrainment was associated with lower

337 oscillation frequencies, as other studies have not reported similar asymmetries ${ }^{13}$.

\section{Metabolic cost of oscillator interaction strategies}

339 Variation in metabolic power was not affected by entrainment but was instead strongly related 340 to net mechanical work done on the subject by the harness tension. Specifically, receiving net 341 positive work was associated with a lower energetic cost than with negative work. This result 342 may be reasonable given: (1) zero net CoM work is required for steady, periodic gait (e.g. net 343 positive oscillator work requires net negative muscle work, and vice versa) and (2) negative 344 muscle work costs 4.8 times less metabolic energy than positive muscle work ${ }^{18}$. This logic 345 assumes that no additional work is needed to compensate for extra force on the body, other 346 than the net negative work needed to manage the energy balance. It is unclear if this 347 assumption holds. 
Ideally, the subject learns a strategy to receive positive power from the oscillator system in such

349 a way that the leg muscles are unburdened from their typical function (e.g. Gordon \& Ferris ${ }^{19}$ ). Indeed, large amounts of positive mechanical power naturally occur due to push off forces during the step-to-step transition in the gait cycle, functioning to redirect the body from falling to rising with the next step ${ }^{20}$. Recent optimization models have identified a strategy for minimizing work while walking with an oscillating impulse applied to the CoM ${ }^{21}$. This strategy aligns upward oscillator forces with the step-to-step transition (motor phase $0^{\circ}$ ). The upward oscillator force does some work that the legs would normally do, thus minimizing cost in the model. Notably, this is a different strategy than what subjects preferred in the current study, where individuals largely aligned oscillation forces with CoM vertical velocity to maximize externally applied positive power (phase $=90^{\circ}$ ). However, the model did not account for differential energetic costs of positive and negative muscle work, nor did it allow for negative work via passive dissipative mechanisms (e.g. collisions during foot-ground contact ${ }^{22-25}$, damping in soft tissue deformation 26,27 , etc.) which require near zero metabolic expenditure.

362 Furthermore, the model did not account for swing leg dynamics and associated costs. These 363 issues and others may be important for understanding subject preferences of increased positive 364 mechanical power from the oscillations.

365 If the preferred strategy uses oscillation forces to replace positive muscle work, then the extent 366 to which this strategy is energetically favorable likely relies on an individual downregulating leg 367 work and associated muscle activity ${ }^{19}$. Yet subjects substantially increased the vertical excursion 368 of their CoM when they entrained to motor oscillations compared to at baseline (an increase of $36958.2 \%$ or $125.5 \%$ for $A_{m}=10 \%$ or $30 \% B W$, respectively). The increased body oscillation is 370 likely evidence that subjects did not downregulate their push off during entrainment. Instead, it 371 seems that subjects preferred to increase positive power from the system by aligning active 372 force peaks approximately with toe off in the step cycle (Fig. 6b, 8a). Furthermore, subjects that 373 leveraged the most positive power from the oscillations had the lowest metabolic cost (local 374 minimum at $\left.\phi \approx 90^{\circ}\right)$. These results, however, should be treated with caution. Experiments 375 presented here did not explicitly control or manipulate the motor phase, but rather, the phase 376 variation depicted in Figure 8a represents subject preferences during the experiment with no 377 metronome. Since subjects did not explore a full range of phase values in steady state, it is 378 unclear if the preferred alignment centers on a global or local optimum. The grey dashed line in 379 Figure 8a indicates a sine wave fit to the data, assuming a cyclical cost over phase. Extrapolation 
of this fit beyond the relatively narrow range of data is questionable, and future studies could control motor phase explicitly to characterize fluctuations in cost.

382 Notably, the data show mostly net negative work in the sampled phase domain, despite

383 maximal positive power expected in this region $\left(\phi \approx 90^{\circ}\right)$. This bias may partially be explained by negative work associated with resistive forces due to reflected inertia of the motors and hardware, as well as damping in the system (e.g. back electromotive force). Increases in vertical excursion of the CoM during entrainment likely contributed to an exaggeration of these effects.

387 Perhaps subjects prefer phase lags that maximize positive mechanical power from the oscillations in part to reduce dissipation in the system.

\section{Interactions with other active devices}

It is possible that the motor control system uses mechanical and/or physiological variables as a proxy for energetic cost, as other researchers have previously suggested ${ }^{28}$. In this case, it appears that subjects prefer to maximize positive mechanical power from the oscillations.

393 However, it is unclear if this preference is generalizable to mechanical interactions with other 394 devices or if it is only relevant to the specific system discussed here. Ahn and Hogan ${ }^{7,8}$ found 395 that subjects aligned ankle torques at push off with those from an ankle exoskeleton. Since the 396 ankle generates high positive power during push off, the alignment likely means that individuals 397 chose to leverage positive power from the device, similar to our subjects. A different study 398 found that individuals prefer to align pulses of electrical stimulation to the plantar flexors either 399 just before toe off or in advance of heel strike ${ }^{5}$.

400 Experiments by Selinger et al. ${ }^{13}$ found subjects adjust their gait in response to resistive damping 401 forces from a knee exoskeleton. While subjects could not possibly receive positive power from 402 the device, they did actively adjust their gait to avoid negative power. Sánchez et al. ${ }^{29}$ 403 investigated gait adaptation of subjects walking on a split-belt treadmill (a treadmill that 404 contains separate belts moving at different speeds for each leg). The authors showed that when 405 subjects are given sufficient time adapting to the system, they employ a step length asymmetry 406 associated with net positive mechanical power from the treadmill and reduced metabolic 407 output. 
These examples provide some evidence of preferred subject interactions that involve either reducing net negative work or increasing net positive work from dynamic external devices. This strategy is consistent with our results that subjects prefer to align motor forces approximately with vertical CoM velocity. However, it is unclear if the ultimate objective is to increase positive power or to decrease metabolic expenditure, since the two are often correlated. Wong et al. ${ }^{30}$ showed that individuals do not adjust gait in exchange for higher levels of oxygen concentration fed to them through an air tube, even as they consciously acknowledge an increased effort from not adjusting their gait. Perhaps individuals are sensitive to positive mechanical power from external sources as an indirect sign of economical interactions with the environment.

\section{Entrainment may stabilize interactions for internal models of gait}

Given that most subjects learned to entrain under a large range of oscillation parameters, it seems reasonable to conclude that individuals largely preferred a stable interaction with the environment in experiments detailed here. This could be interpreted as evidence of a feedforward gait control mechanism since unpredictability could impede effective implementation of internal models. Various studies have shown evidence of a dual-part locomotor control process, including a rapid response to external stimuli and a slower, more gradual, fine-tuning of the response $\mathrm{e}^{31-33}$. These findings are interpreted as evidence of an internal model used to make quick predictions (within seconds) regarding energetic cost based on state estimations and followed up with direct energy optimization occurring more slowly (within minutes). Other researchers have proposed that feedforward and feedback control mechanisms also play a role during gait adaptation to split-belt treadmills ${ }^{34}$ and lateral perturbation systems used to train individuals with incomplete spinal cord injury ${ }^{35}$.

The experiments presented in the current study describe subject interactions that can be relatively volatile, at least before subjects converge on entrainment. In particular, inexperience with the oscillation system may require a prerequisite to the dual-part control of locomotion described previous: a stabilization phase. Here, stability does not necessarily refer to fall avoidance or balance, but rather to a state of relative consistency, where interactions with the environment are sufficiently repeatable over subsequent steps. A relatively stable interaction may be required before feedforward or feedback control can be successfully implemented, and entrainment could provide that stability. 
438 Koban et al. ${ }^{36}$ explained a similar perspective in a slightly different context, with regards to 439 individuals that entrain gait when walking side by side (i.e. interpersonal synchronization). They 440 described a process by which entrainment occurs to reduce the perceived mismatch between an 441 expectation about their companion's motor behavior based on their own. An analogous

442 principle could be adapted to the expectation of mechanical interactions with the environment, 443 as mediated by the entrainment opportunities associated with the oscillator system described in 444 this manuscript. In lieu of a direct metabolic motivation for entrainment, it may be possible that 445 the motor control system prefers a relatively stable interaction with the environment so as to 446 make feedforward predictions more precise and actionable.

\section{Methods}

\section{Participants}

449 A convenience sample of ten healthy university students (five males, five females) were 450 recruited. The mean [ \pm 1 standard deviation (SD)] subject height was $1.71 \pm 0.07 \mathrm{~m}$, leg length 451 was $0.91 \pm 0.06 \mathrm{~m}$, weight was $65.7 \pm 12.2 \mathrm{~kg}$ and age was $26.2 \pm 2.9$ years. Exclusion criteria for 452 the experiments included any musculoskeletal injuries or neurological conditions affecting one's 453 gait or their ability to carry a heavy backpack. All participants provided informed consent to 454 participate, and these studies were approved by an ethics review board at the University of 455 Calgary (REB16-1517). All methods were performed in accordance with the relevant guidelines 456 and regulations.

\section{Measurements and analysis}

458 Inertial measurement units, or IMUs (Xsens Technologies B.V., Enschede, The Netherlands) were 459 placed at each ankle and the lower back. The ankle sensors were used to detect signal peaks and 460 calculate step frequency as the inverse of the time period between peaks. The back sensor 461 provided kinematics to approximate CoM motion (acquisition rate $=100 \mathrm{~Hz}$ ). These data were 462 integrated twice over time to get velocity and displacement, and a moving average (window set 463 to stride time) was subtracted from the signal to adjust for low frequency drift error. A moving 464 average filter (window of \pm 5 steps at each data point) was also applied to step frequency 465 measurements to more clearly illustrate trends over time. Step frequency $\left(f_{s}\right)$ was normalized 
by the subject's baseline frequency $\left(f_{p}\right)$ measured while walking in the system with the motors inactive.

$$
f_{r}=\frac{f_{s}}{f_{p}}
$$

Custom in-line tension transducers were built with strain gauges (Micro-Measurements CEA-06125UW-350, Wendell, NC, USA) configured in half-bridge circuits. The strain gauges were

471 epoxied to C-shaped steel hooks and measured tension in cables pulling on the body harness

472 during experiments (Fig. 1). The strain gauge signals were passed to a strain conditioning

473 amplifier (National Instruments, SCXI-1000 with SCXI-1520 eight-channel universal strain gauge

474 module connected with SCXI-1314 terminal block, Austin, Texas USA), digitized (NI-USB-6251

475 mass termination) and acquired in a custom virtual instrument in LabVIEW (National

476 Instruments) at an acquisition rate of $100 \mathrm{~Hz}$. The transducers were calibrated with known

477 weights before every testing session using a least squares linear regression to quantify a

478 conversion factor from volts to Newtons force $\left(R^{2}>0.99\right)$.

479 At the beginning of each trial, a few seconds of data were collected where the subject stood still 480 before starting the treadmill. During this time, the transducer signals measured force from the 481 motors' weight in the system plus nominal tension from the motors pulling the system taut. This 482 initial tension was averaged over a ten second interval and subtracted from the subsequent 483 signal in the trial.

484 Next, tension forces were multiplied by vertical velocity to calculate mechanical power acting on 485 the CoM. Step cycles were distinguished by peaks in the vertical acceleration of the CoM 486 (approximately indicating the middle of double stance). Tension forces, kinematics and 487 mechanical power were all segmented into blocks of data comprising every step cycle identified 488 in all trials. These data were interpolated at regular intervals matching the average resolution of 489 the raw data collection ( $\sim 55$ data points per step).

490 Pulse signals were recorded in LabVIEW to mark the timing of peak current sent to the motors 491 relative to the start time of a given step cycle $\left(\Delta t_{p}\right)$. In addition to data synchronization, the 492 pulse signals were used to calculate the phase lag of peak current relative to the step cycle $(\phi)$.

$$
\phi=\Delta t_{p} f_{s} 360^{\circ}
$$


494 Due to dynamics of the system, there was a slight delay from when peak current was driven to 495 the motors to when tension spiked in the harness. The average delay, $\Delta \phi$, was calculated for all 496 subjects and trials and phase data were shifted as appropriate: $\phi^{*}=\phi+\Delta \phi$.

497 Oxygen consumption and carbon dioxide elimination rates were measured using a commercial 498 metabolic analysis system (TrueMax 2400, ParvoMedics, Salt Lake City, UT, USA). All trials lasted 499 longer than five minutes, which allowed metabolic data to reach steady state conditions before 500 mean and SD values were calculated (typically the remaining 2-3 minutes of each trial). Oxygen 501 consumption rates in $\mathrm{ml} \mathrm{O}_{2} \mathrm{~S}^{-1}$ were multiplied by a factor of 20.1 to convert to Watts. Gross 502 metabolic rate was converted to net metabolic rate by subtracting baseline values during quiet 503 standing. Net metabolic power was non-dimensionalized by dividing belt speed and subject BW 504 (sometimes referred to as non-dimensional cost of transport). During all metabolics testing, the 505 data were deemed acceptable if the respiratory exchange ratio remained below a value of 1.0.

\section{Test protocol}

507 During Baseline 1, subjects walked on the treadmill freely (i.e. without the body harness; Fig. 2b) 508 for a five-minute duration. The treadmill speed $\left(v_{b}=1.19 \mathrm{~m} \mathrm{~s}^{-1}\right.$ on average) was programmed 509 such that non-dimensional speed, $\tilde{v}$, equaled 0.4 during all testing.

$$
v_{b}=\tilde{v} \sqrt{g L}
$$

511 where $L$ is the subject's hip height measured from the ground while standing. Speed was

512 rounded to the nearest tenth of a $\mathrm{km} /$ hour, per the treadmill's available resolution.

513 During Baseline 2, subjects walked while wearing the body harness connected to the pulley514 cable system (Fig. 2b) for a ten-minute duration. Both actuators provided a constant nominal 515 tension (approximately 10\% BW) to reduce slack in the system, but the average net force on the 516 body was zero since one cable pulled up while the others pulled down. Subjects also

517 experienced added inertia from the motors and associated hardware connected in the system, 518 as well as friction and damping. Each subject's baseline preferred step frequency $\left(f_{p}\right)$ was 519 assessed and motor frequencies prescribed in testing conditions were determined relative to 520 this baseline. 
521 During experiments, subjects walked in the harness for two minutes with nominal motor current

522 to avoid cable slack. Next, current oscillations were commanded to the motors at a constant

523 frequency and amplitude, and the subject was instructed to respond freely (Fig. 2a). Five

524 minutes later, subjects were asked to step to the beep of a metronome (matched to their

525 baseline frequency, i.e. "frequency clamping"; Fig. 2a) even as oscillations continued at a

526 different frequency. After another five minutes, the oscillations and metronome ceased, and the

527 subject prepared to end the trial. Metabolic data was collected throughout this test to compare

528 oxygen consumption while responding freely in the system (allowed to entrain) versus walking

529 to the metronome (not allowed to entrain). This test was performed with various oscillation

530 amplitudes $\left(A_{m}=10,30 \% B W\right)$ and motor frequencies relative to baseline step frequency

$531\left(\Delta f_{m}=0, \pm 6 \%\right.$; see Fig. $\left.2 \mathrm{~b}\right)$.

532

$$
\Delta f_{m}=\frac{f_{m}-f_{p}}{f_{p}} 100 \%
$$

533 In the case of trial conditions where $\Delta f_{m}=0 \%$, no metronome was played and the trial ended

534 after five minutes of oscillations. Trial conditions were randomized to minimize any ordering 535 effects.

536 During experiments, a curtain was used to blind subjects from any motion of the pulleys or 537 motors (Fig. 1b). Ambient noise was played through headphones to help block out rhythmic 538 sounds of the system. During trials, subjects were asked to walk in any manner that felt most 539 natural or extracted minimal effort. However, subjects were encouraged to explore different 540 aspects of their gait, including stride length. Note, a more detailed description of the oscillator 541 system design and operation can be found in the Supplementary Materials.

\section{Defining entrainment}

543 Entrainment was defined with arbitrary thresholds: any step frequencies within \pm 3 SDs

$544(\sim \pm 0.02 \mathrm{~Hz})$ of the prescribed motor frequency for at least sixteen out of twenty $(80 \%)$

545 consecutive steps. The SD of subjects was determined from the last minute of data from

546 Baseline 2 (treadmill walking with the harness). Two metrics quantified the level of entrainment

547 for a subject in each trial. The entrainment step ratio $(E S R)$ is the ratio of entrained steps to 548 total steps taken during experiment (without the metronome) while the average duration of 
entrainment $\left(\Delta \bar{t}_{e}\right)$ was used to estimate entrainment durations since subjects sometimes drifted

550 in and out of the motor's frequency.

\section{Statistical analysis}

552 Filtered relative step frequency data were interpolated at equal time intervals matching the 553 data acquisition rate. Median values of the interpolated data were taken across all subjects who 554 entrained at least once in the trial and at each time point, to represent skewed distributions 555 more appropriately. Quartiles characterized the spread of the distribution for each time point at $556 \quad 25 \%$ and $75 \%$ levels.

557 Linear mixed models were used to assess various outcomes during experiments. The mixed 558 model was chosen to control for repeated measurements among subjects participating in 559 multiple trials each; subject was included in the models as a random effect. All statistical models 560 were developed and evaluated in JMP (SAS Institute Inc., Cary, NC USA, version 14.1.0) using the 561 restricted maximum likelihood method for parameter estimation and a compound symmetric 562 covariance structure.

563 In three models, motor frequency $\left(\Delta f_{m}\right)$ and amplitude $\left(A_{m}\right)$ as well as an interaction between 564 the two $\left(\Delta f_{m} \times A_{m}\right)$ were added as fixed effects to test if the oscillation parameters contributed 565 significantly to the various outcomes. The first two models tested the effect of oscillation 566 parameters on the level of entrainment via $\operatorname{ESR}$ and $\Delta \bar{t}_{e}$.

567 In order to assess metabolic power, a linear mixed model was stratified by trial condition,

568 baseline type (wearing or not wearing the harness) and the metronome's status (i.e. active or 569 inactive) during data collection. A post hoc Tukey's Honestly Significant Difference test was used 570 to detect differences in estimates of metabolic power $(\alpha=0.05)$, while controlling for multiple

571 hypothesis testing. Given the metronome's status only indicates whether an individual has the 572 capacity to entrain and not whether they actually did entrain, a separate model was used to test 573 for the effect of entrainment level (via entrainment step ratio, ESR) as a covariate for metabolic 574 power. $\Delta \bar{t}_{e}$ was not included to avoid collinearity. Mechanical work done by the harness tension 575 was also included to assess any effect of the mechanical interaction on cost.

576 The significance of fixed model effects was evaluated with $95 \%$ confidence limits and post hoc 577 t-tests where $p$ values were adjusted $\left(p_{a d j}\right)$ using the Bonferroni correction depending on how 
578 many tests were performed in the model. Tests were considered significant if $p_{a d j}<0.05$.

579 Throughout the manuscript, unadjusted $p$ values are reported, and significance is indicated with

580 asterisks. A summary of statistical model results is found in the Supplementary Materials.

581

582

\section{References}

584 1. Dallard, P. et al. London Millennium Bridge: Pedestrian-induced lateral vibration. Journal $585 \quad$ of Bridge Engineering 6, 412-417 (2001).

586 2. Peters, B. T., Brady, R. A. \& Bloomberg, J. J. Walking on an oscillating treadmill: Strategies 587 of stride-time adaptation. Ecological Psychology 24, 265-278 (2012).

588 3. Nessler, J. A., Heredia, S., Bélair, J. \& Milton, J. Walking on a vertically oscillating 589 treadmill: Phase synchronization and gait kinematics. PLOS ONE 12, e0169924 (2017).

590 4. Tackett, E. The effect of noise on gait synchronization to a vertical oscillating treadmill. $591 \quad$ (2018).

592 5. Thorp, J. E. \& Adamczyk, P. G. Mechanisms of gait phase entrainment in healthy subjects 593 during rhythmic electrical stimulation of the medial gastrocnemius. PLOS ONE 15, $594 \quad$ e0241339 (2020).

595 6. Goldfield, E. C., Kay, B. A. \& Warren, W. H. Infant bouncing: The assembly and tuning of 596 action systems. Child Development 64, 1128-1142 (1993).

597 7. Ahn, J. \& Hogan, N. Feasibility of dynamic entrainment with ankle mechanical 598 perturbation to treat locomotor deficit. in 2010 Annual International Conference of the 599 IEEE Engineering in Medicine and Biology Society, EMBC'10 3422-3425 (2010). 600 doi:10.1109/IEMBS.2010.5627892.

601 8. Ahn, J. \& Hogan, N. Walking is not like reaching: Evidence from periodic mechanical 602 perturbations. PLoS ONE 7, e31767 (2012). 
603

604

605

606

607

608

609

610

611

612

613

614

615

616

617

618

619

620

621

622

623

624

625

626

627

628

629

9. Donelan, M., Kram, R. \& Kuo, A. Mechanical and metabolic determinants of the preferred step width in human walking. Proceedings of the Royal Society B: Biological Sciences 268, 1985-1992 (2001).

10. Bertram, J. E. A. Constrained optimization in human walking: cost minimization and gait plasticity. Journal of Experimental Biology 208, 979-991 (2005).

11. Bertram, J. E. A. \& Ruina, A. Multiple walking speed-frequency relations are predicted by constrained optimization. Journal of Theoretical Biology 209, 445-453 (2001).

12. Kuo, A. D. A simple model of bipedal walking predicts the preferred speed-step length relationship. Journal of Biomechanical Engineering 123, 264-269 (2001).

13. Selinger, J. C., O'Connor, S. M., Wong, J. D. \& Donelan, J. M. Humans can continuously optimize energetic cost during walking. Current Biology 25, 2452-2456 (2015).

14. Selinger, J. C., Wong, J. D., Simha, S. N. \& Donelan, J. M. How humans initiate energy optimization and converge on their optimal gaits. Journal of Experimental Biology 222, jeb198234 (2019).

15. Simha, S. N., Wong, J. D., Selinger, J. C. \& Donelan, J. M. A mechatronic system for studying energy optimization during walking. IEEE Transactions on Neural Systems and Rehabilitation Engineering 27, 1416-1425 (2019).

16. Croft, J. L., Schroeder, R. T. \& Bertram, J. E. A. The goal of locomotion: Separating the fundamental task from the mechanisms that accomplish it. Psychonomic Bulletin and Review 24, 1675-1685 (2017).

17. Wong, J. D., Selinger, J. C. \& Donelan, J. M. Is natural variability in gait sufficient to initiate spontaneous energy optimization in human walking? Journal of Neurophysiology 121, 1848-1855 (2019).

18. Margaria, R. Positive and negative work performances and their efficiencies in human locomotion. Int. Z. angew. Physiol. einschl. Arbeitsphysiol 25, 339-351 (1968).

19. Gordon, K. E. \& Ferris, D. P. Learning to walk with a robotic ankle exoskeleton. Journal of Biomechanics 40, 2636-2644 (2007). 
20. Donelan, J. M., Kram, R. \& Kuo, A. D. Simultaneous positive and negative external mechanical work in human walking. 35, 117-124 (2002).

21. Schroeder, R. T. \& Bertram, J. E. Minimally actuated walking: Identifying core challenges to economical legged locomotion reveals novel solutions. Frontiers in Robotics and A/ 5, (2018).

22. Ruina, A., Bertram, J. E. A. \& Srinivasan, M. A collisional model of the energetic cost of support work qualitatively explains leg sequencing in walking and galloping, pseudoelastic leg behavior in running and the walk-to-run transition. Journal of Theoretical Biology 237, 170-192 (2005).

23. Bertram, J. E. A. \& Hasaneini, S. J. Neglected losses and key costs: Tracking the energetics of walking and running. Journal of Experimental Biology 216, 933-938 (2013).

24. Kuo, A. D. Energetics of actively powered locomotion using the simplest walking model. Journal of Biomechanical Engineering 124, 113-120 (2002).

25. Lee, D. v, Comanescu, T. N., Butcher, M. T. \& Bertram, J. E. A. A comparative collisionbased analysis of human gait. Proceedings of the Royal Society B: Biological Sciences 280, 20131779 (2013).

26. Zelik, K. E. \& Kuo, A. D. Human walking isn't all hard work: Evidence of soft tissue contributions to energy dissipation and return. Journal of Experimental Biology 213, 4257-4264 (2010).

27. Riddick, R. C. \& Kuo, A. D. Soft tissues store and return mechanical energy in human running. Journal of Biomechanics 49, 436-441 (2016).

28. Snaterse, M., Ton, R., Kuo, A. D. \& Donelan, J. M. Distinct fast and slow processes contribute to the selection of preferred step frequency during human walking. Journal of applied physiology (Bethesda, Md. : 1985) 110, 1682-90 (2011).

29. Sánchez, N., Simha, S. N., Donelan, J. M. \& Finley, J. M. Taking advantage of external mechanical work to reduce metabolic cost: the mechanics and energetics of split-belt treadmill walking. The Journal of Physiology 597, 4053-4068 (2019). 
30. Wong, J. D., O'connor, S. M., Selinger, J. C. \& Donelan, J. M. Contribution of blood oxygen and carbon dioxide sensing to the energetic optimization of human walking. Journal of Neurophysiology 118, 1425-1433 (2017).

31. Snaterse, M., Ton, R., Kuo, A. D. \& Maxwell Donelan, J. Distinct fast and slow processes contribute to the selection of preferred step frequency during human walking. Journal of Applied Physiology 110, 1682-1690 (2011).

32. O'Connor, S. M. \& Donelan, J. M. Fast visual prediction and slow optimization of preferred walking speed. Journal of Neurophysiology 107, 2549-2559 (2012).

33. Pagliara, R., Snaterse, M. \& Donelan, J. M. Fast and slow processes underlie the selection of both step frequency and walking speed. Journal of Experimental Biology 217, 29392946 (2014).

34. Mawase, F., Haizler, T., Bar-Haim, S. \& Karniel, A. Kinetic adaptation during locomotion on a split-belt treadmill. Journal of Neurophysiology 109, 2216-2227 (2013).

35. Wu, M., Brown, G. \& Gordon, K. E. Control of locomotor stability in stabilizing and destabilizing environments. Gait \& Posture 55, 191-198 (2017).

36. Koban, L., Ramamoorthy, A. \& Konvalinka, I. Why do we fall into sync with others? Interpersonal synchronization and the brain's optimization principle. Social Neuroscience 14, 1-9 (2019).

\section{Acknowledgments}

This work was supported by a Natural Sciences and Engineering Research Council of Canada (NSERC) Discovery grant (312117-2012 and 04823-2017) received by J.E.A.B., a University of Calgary Eyes High Doctoral Recruitment Scholarship (EHDRS) received by R.T.S., and a Biomedical Engineering Equipment Grant received by R.T.S. In addition, the authors would like to thank Gregory Sawicki, Vinzenz von Tscharner, and Nigel Shrive for their helpful comments and discussion regarding this work. 


\section{Author contributions}

685

The authors declare no competing interests.

\section{Competing interests}

a.

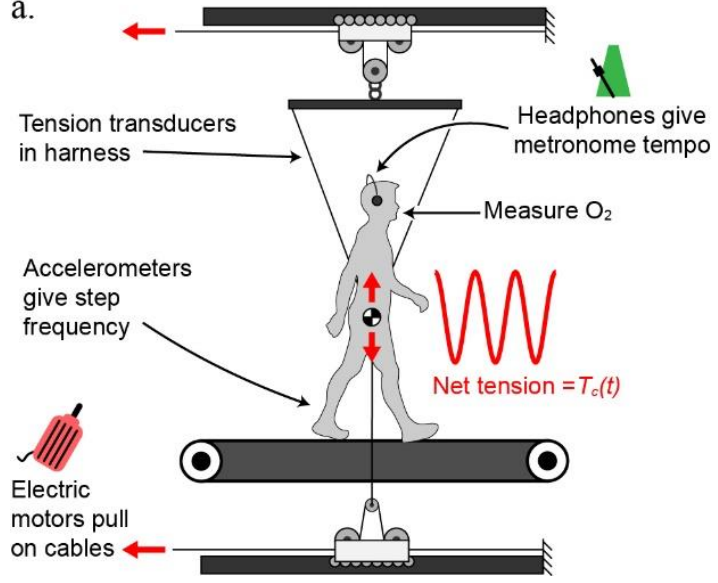

b.

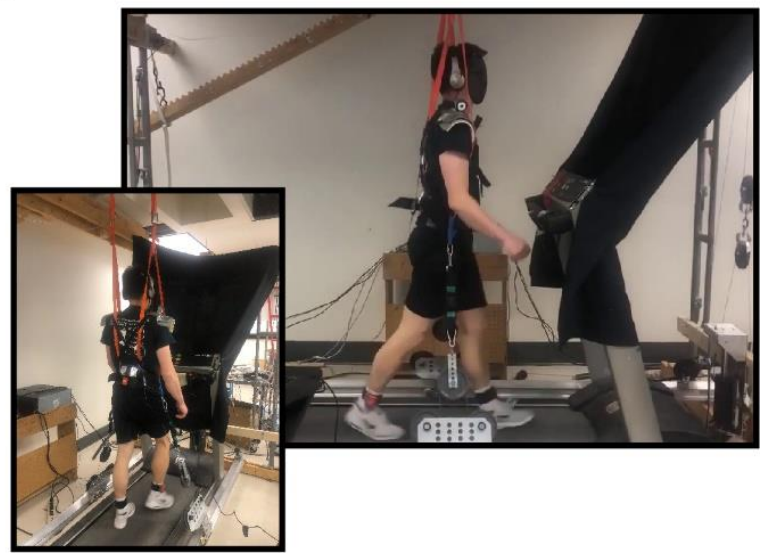

Figure 1. System schematic and images. (a) A schematic of the oscillator system is depicted in the sagittal plane. (b) Images of a subject walking in the system during a trial, from a side view

697 and from behind. Downward force came as the resultant of self-equalizing oblique cables. A curtain was used to blind the subject from any motion of the pulleys or motors, and headphones were used to play ambient noise so as to block out rhythmic sounds from the system. The

700 headphones were also used to play a metronome beep during portions of the experiment.

701 Additional details regarding the oscillator system design and operation can be found in the 702 Supplementary Materials. 
a.

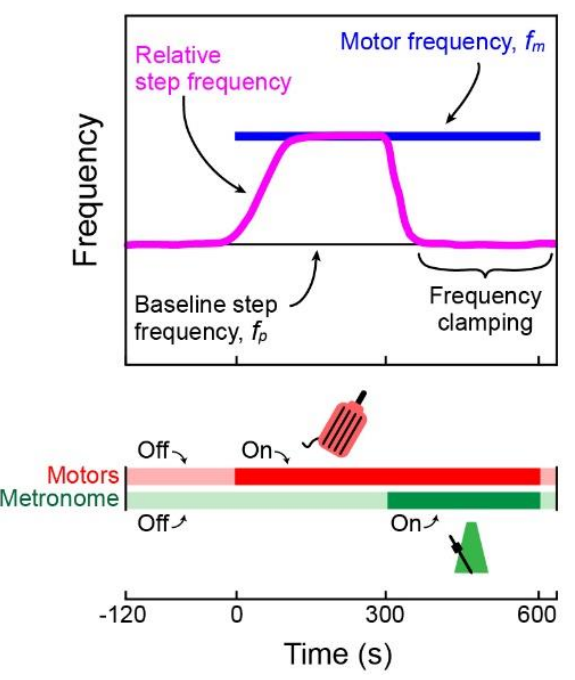

b.
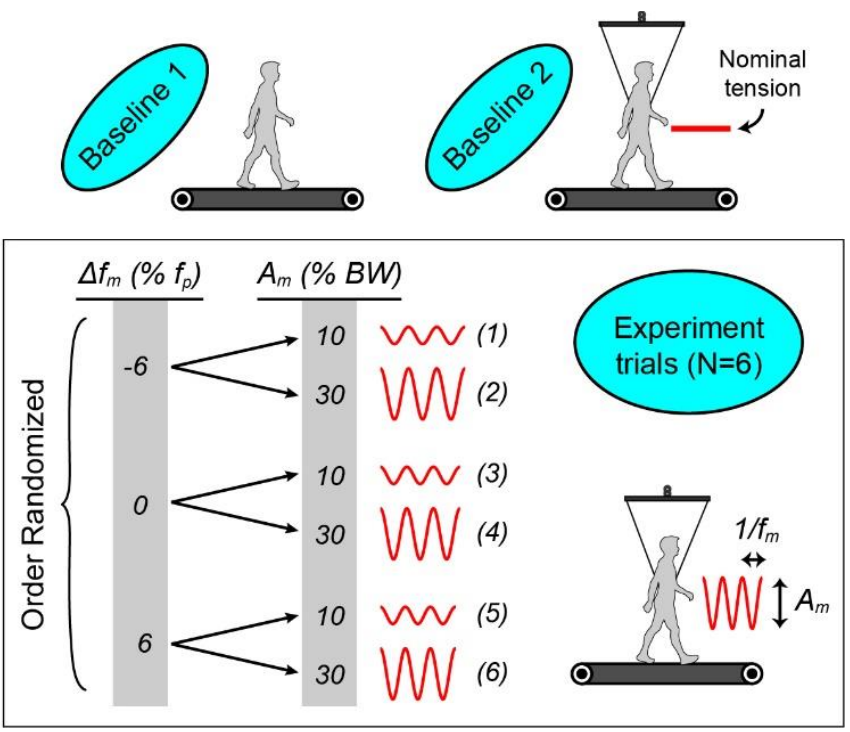

704

705

706

707

708

709

710

711

712

713

714

715

716

Figure 2. Experimental protocol. (a) A generic trial condition is depicted with simulated step frequency data (magenta) over time and constant motor frequency (blue). There are no oscillations during the first two minutes of the test. Motor oscillations begin at time zero and continue for five minutes while subjects freely interact with the system. Next, oscillations continue for another five minutes and the metronome directs individuals to step at their baseline preferred frequency $\left(f_{p}\right)$ despite the external oscillation frequency ("frequency clamping"). The oscillations and metronome are terminated, and subject is given fifteen additional seconds to approach the end of the trial. (b) Baseline conditions and oscillation parameters during experiment trials are shown: $\Delta f_{m}=0, \pm 6 \%$ and $A_{m}=10,30 \%$ body weight (BW). Trial conditions were implemented randomly to reduce ordering effects. 

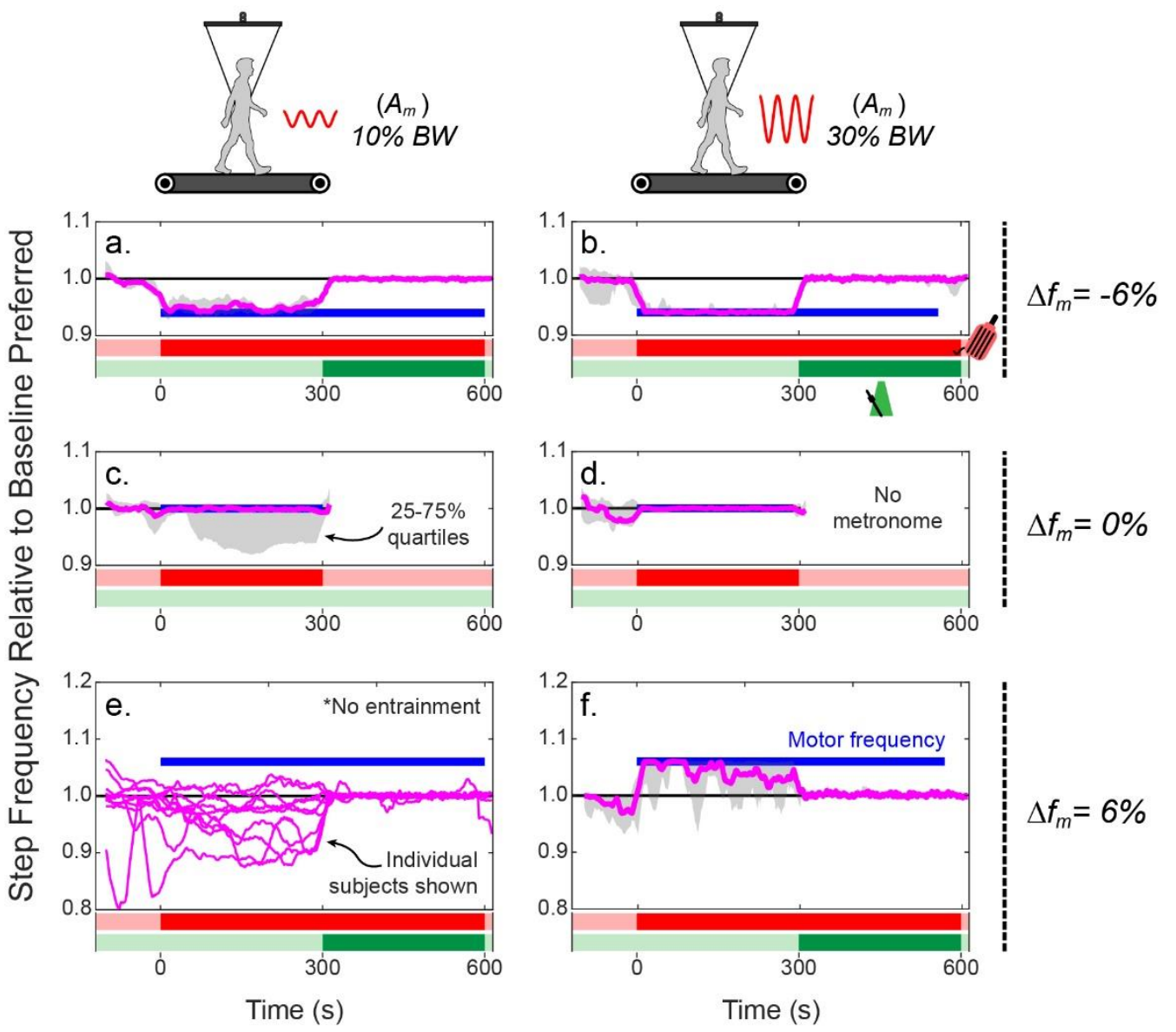

718 Figure 3. Entrainment results. The median relative step frequency ( $f_{r}$, step frequency divided by 719 preferred step frequency in Baseline 2; magenta) of all subjects who entrained is plotted over 720 the trial duration. $25 \%$ and $75 \%$ quartiles are used to indicate the distribution at every time 721 point (grey shaded area). All trial conditions are shown, including: $\Delta f_{m}=-6 \%$ in (a) and (b);

$722 \Delta f_{m}=0 \%$ in (c) and (d); $\Delta f_{m}=6 \%$ in (e) and (f); $A_{m}=10 \% B W$ in (a), (c) and (e); $A_{m}=$

$72330 \% B W$ in (b), (d) and (f). The oscillations began at Time $=0 s$ and ended at approximately 724 Time $=600 \mathrm{~s}$. During $0 \leq$ Time $\leq 300 \mathrm{~s}$, subjects responded freely to the force oscillations. 725 During $300 \leq$ Time $\leq 600 s$, subjects were directed to follow the cadence of the metronome 726 at their predetermined baseline step frequency ("frequency clamping") even as the oscillations 727 continued at a different frequency. There was no metronome used in trials where $\Delta f_{m}=0$, 728 since frequencies were already matched. As a result, these experiments ended after around 729 Time $=300 \mathrm{~s}$. Note, median data are only shown for individuals who entrained at least once 730 throughout the trial. In the trial condition where $\Delta f_{m}=6 \%$ and $A_{m}=10 \% B W$, individual 731 subject data are shown instead since no entrainment occurred. 
a.

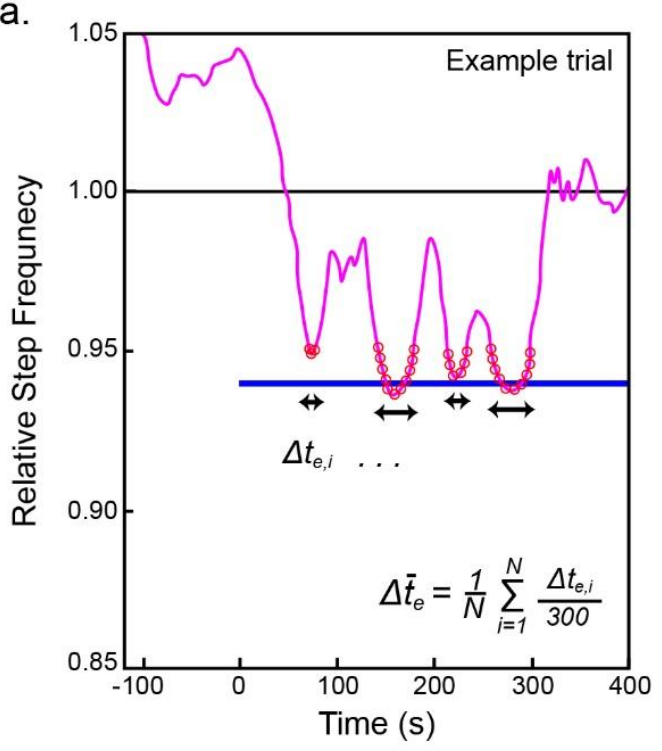

b.
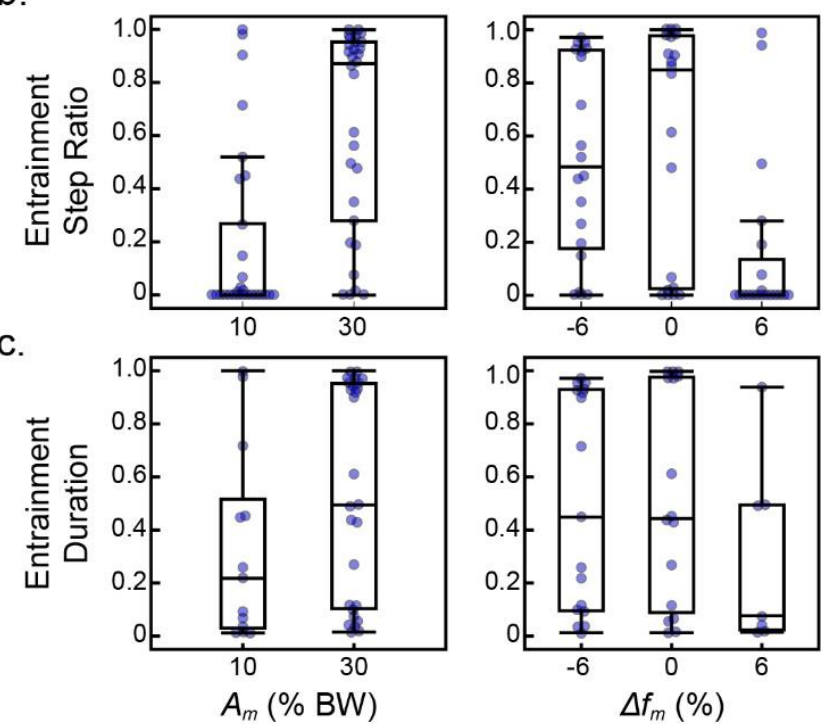

733 Figure 4. Entrainment is often transient. (a) Data from an example subject illustrates transient

734 entrainment where relative step frequency (magenta) oscillates towards and away from the

735 motor frequency (blue). Red data points indicate when the subject is considered entrained with

736 the oscillator system (see Methods section for details on entrainment definition). (b) The

737 entrainment step ratio ( $E S R$; ratio of entrained steps to total steps taken during the first five

738 minutes of oscillations in the experiment) and (c) the average entrainment duration ( $\Delta \bar{t}_{e}$;

739 average time duration of bouts of entrainment) are shown as a function of oscillation amplitude

740 and motor frequency, where each data point represents a subject's level of entrainment during

741 each trial and box plots summarize the distribution. Linear mixed models were used to

742 statistically test the effects of trial conditions on both entrainment metrics shown here (see

743 Table S1 in the Supplementary Materials for full results). 


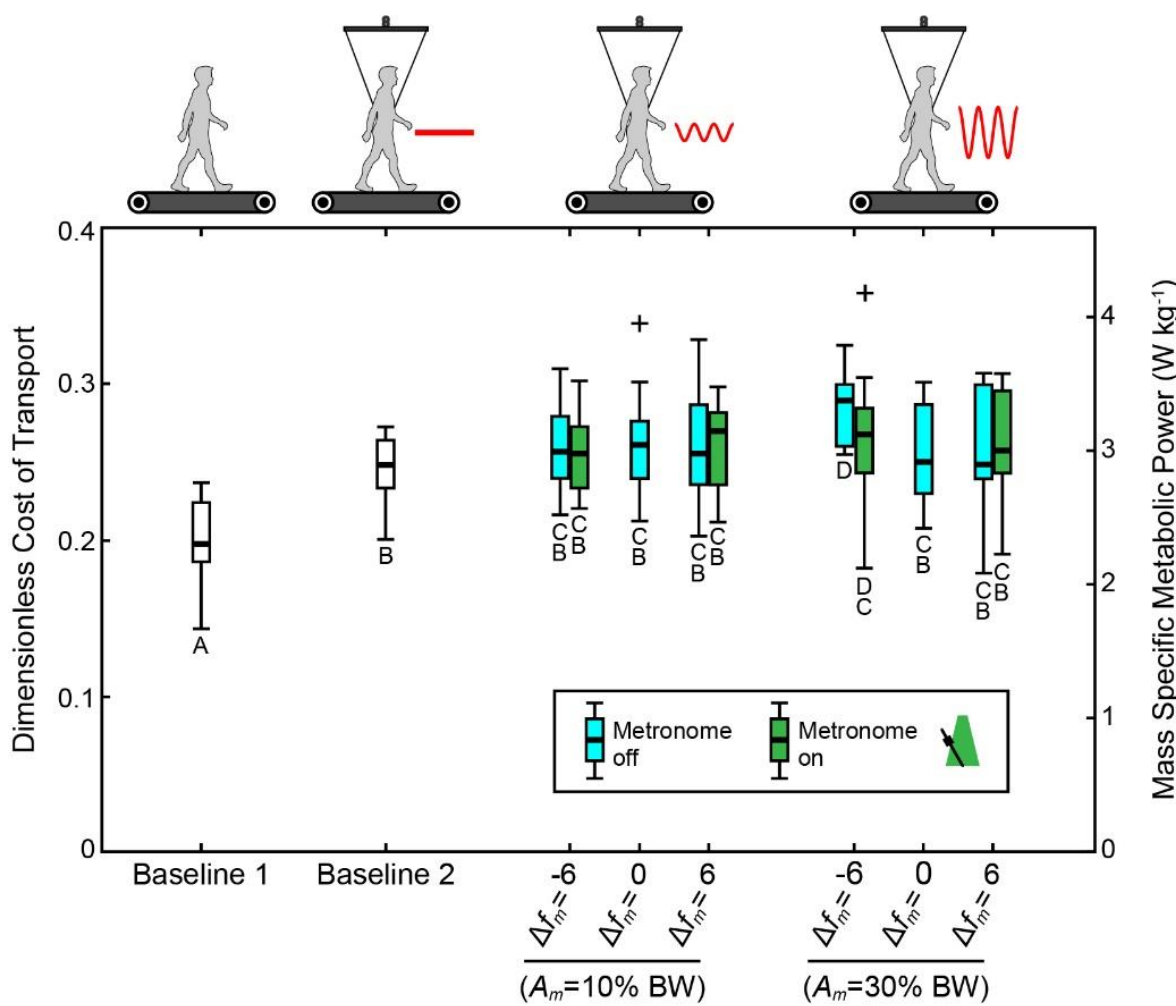

746 Figure 5. Metabolic power does not depend on entrainment. Non-dimensional metabolic power

747 is compared between all trial conditions and baseline tests. A Tukey Honestly Significant

748 Difference test was performed on least squares mean values for all conditions. Box plots are

749 labelled with letters indicating conditions where power is not significantly different. Outliers are

750 marked with " + ". See Table S2 in the Supplementary Materials for full results. Baseline 1 and 2

751 refer to walking on the treadmill without and with the harness, respectively. Box plots are

752 shown for all metabolic data collected during the first five minutes of oscillations where the

753 subject freely responded to the oscillation forces (i.e. metronome off). During the next five

754 minutes of oscillations, a metronome guided subjects to step at their baseline preferred

755 frequency (importantly, not matched to the oscillation frequency). This allowed for metabolic

756 cost comparisons between entrained and non-entrained gait. 

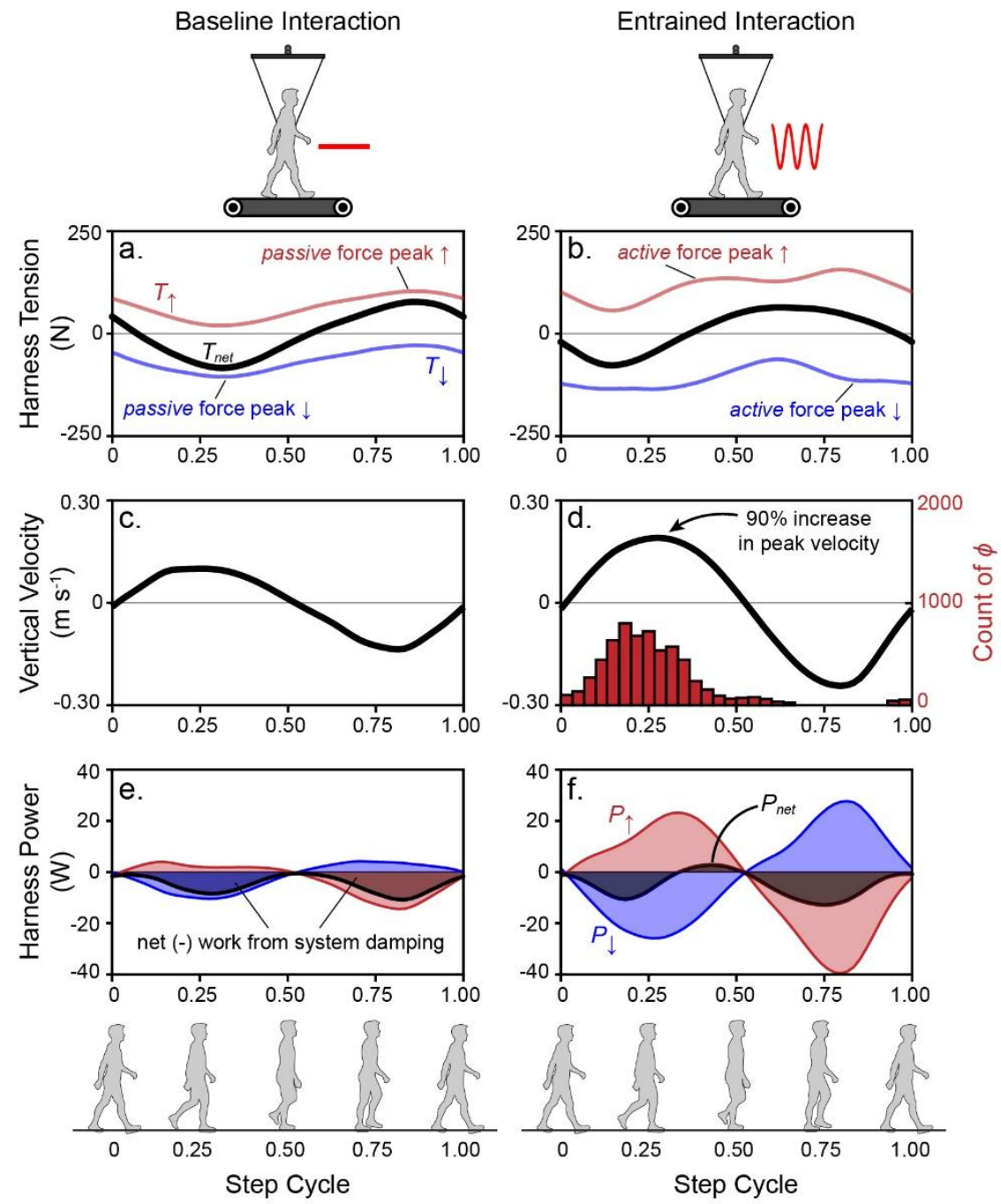

Figure 6. Subjects prefer to align peak oscillation forces at toe off. The average harness tension

759 is shown in (a) and (b), where the red and blue curves indicate cable tension pulling up ( $\left.T_{\uparrow}\right)$ and down $\left(T_{\downarrow}\right)$, respectively, and black indicates net tension $\left(T_{n e t}\right)$. Average center of mass vertical velocity is shown in (c) and (d), while the red histogram in (d) indicates the distribution of motor phase chosen by subjects during entrainment. Average power from the cables pulling up $\left(P_{\uparrow}\right.$, red) and down $\left(P_{\downarrow}\right.$, blue) are shown in (e) and (f), while net power $\left(P_{n e t}\right)$ is indicated with black shading. Panels (a), (c) and (e) indicate average curves measured during Baseline 2 (walking with the harness but no active oscillations), while panels (b), (d) and (f) indicate average curves measured during experiment trials. Snapshots of the walking step cycle are shown near the bottom to help orient the reader to the timing of events shown in plots. Zero in the step cycle corresponds with double stance while 0.5 corresponds with single stance. 

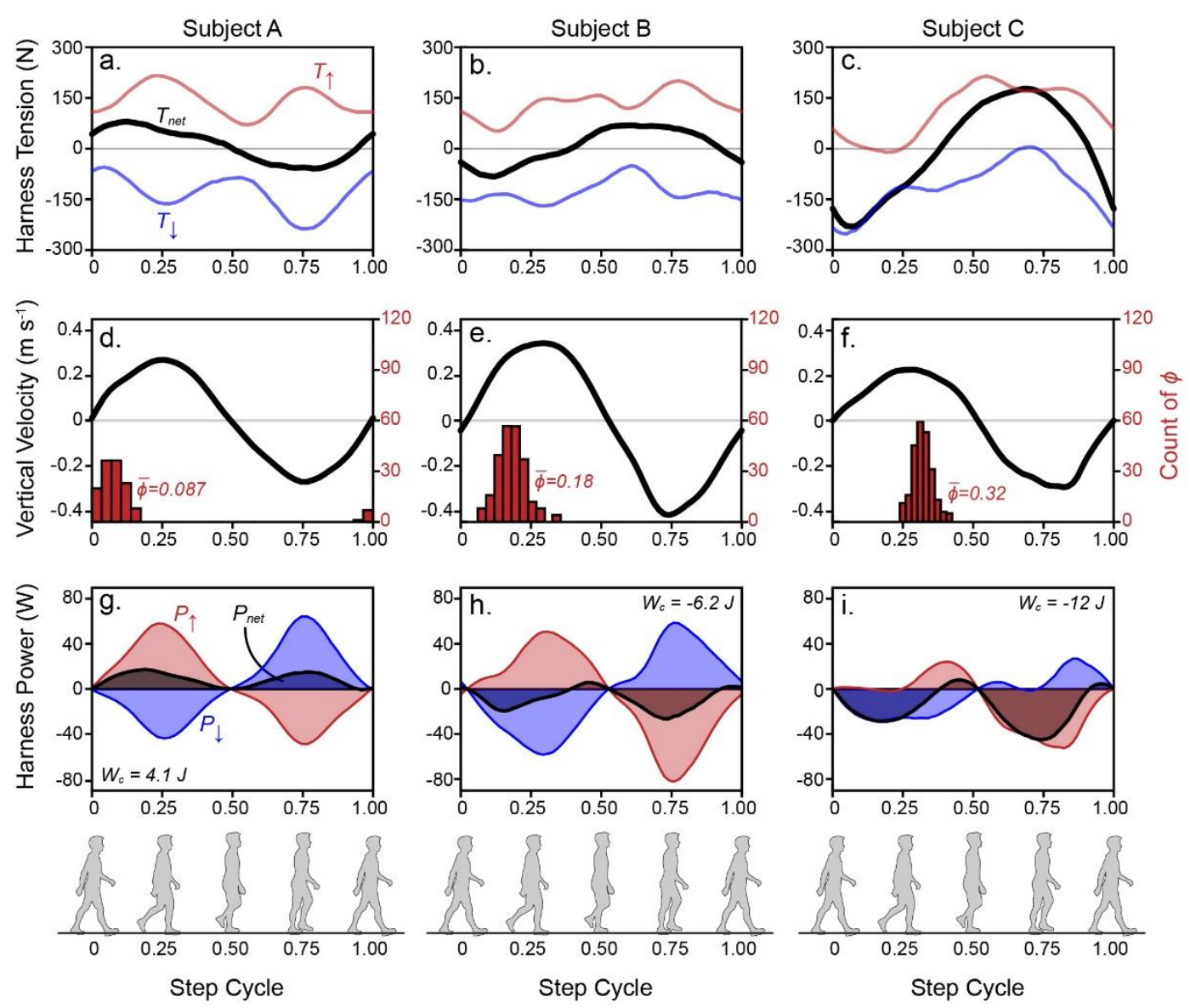

Figure 7. Subjects prefer a range of phase alignments, resulting in variable net power. Data

771 from three subjects were chosen to demonstrate varying entrainment strategies. Average data

772 for Subject A ( $\Delta f_{m}=6 \%, A_{m}=30 \% B W, 297$ steps averaged) are shown in (a), (d) and (g),

773 where motor phase alignment occurs at $\bar{\phi}=0.087$ of the gait cycle. This strategy aligns peak

774 tension forces with the center of mass (CoM) vertical velocity and thus, results in net positive

775 work $\left(W_{c}=4.1 \mathrm{~J}\right)$. Average data for Subject B $\left(\Delta f_{m}=-6 \%, A_{m}=30 \% B W, 475\right.$ steps

776 averaged) are shown in (b), (e) and (h) where motor phase alignment occurs at $\bar{\phi}=0.18$ of the

777 gait cycle. Since peaks in tension due to motor forces occur slightly later, positive power is

778 relatively low and net negative work occurs $\left(W_{c}=-6.2 \mathrm{~J}\right)$. Average data for Subject $\mathrm{C}\left(\Delta f_{m}=\right.$

$779-6 \%, A_{m}=30 \% B W, 444$ steps averaged) are in (c), (f) and (i) where $\bar{\phi}=0.32$ of the gait

780 cycle, and mechanical power from the harness tension is dominated by resistive inertial forces,

781 thus leading to substantial net negative work on the $\operatorname{CoM}\left(W_{c}=-12 \mathrm{~J}\right)$. Data points for subjects

782 A, B and C are labelled in Figure 8b. 

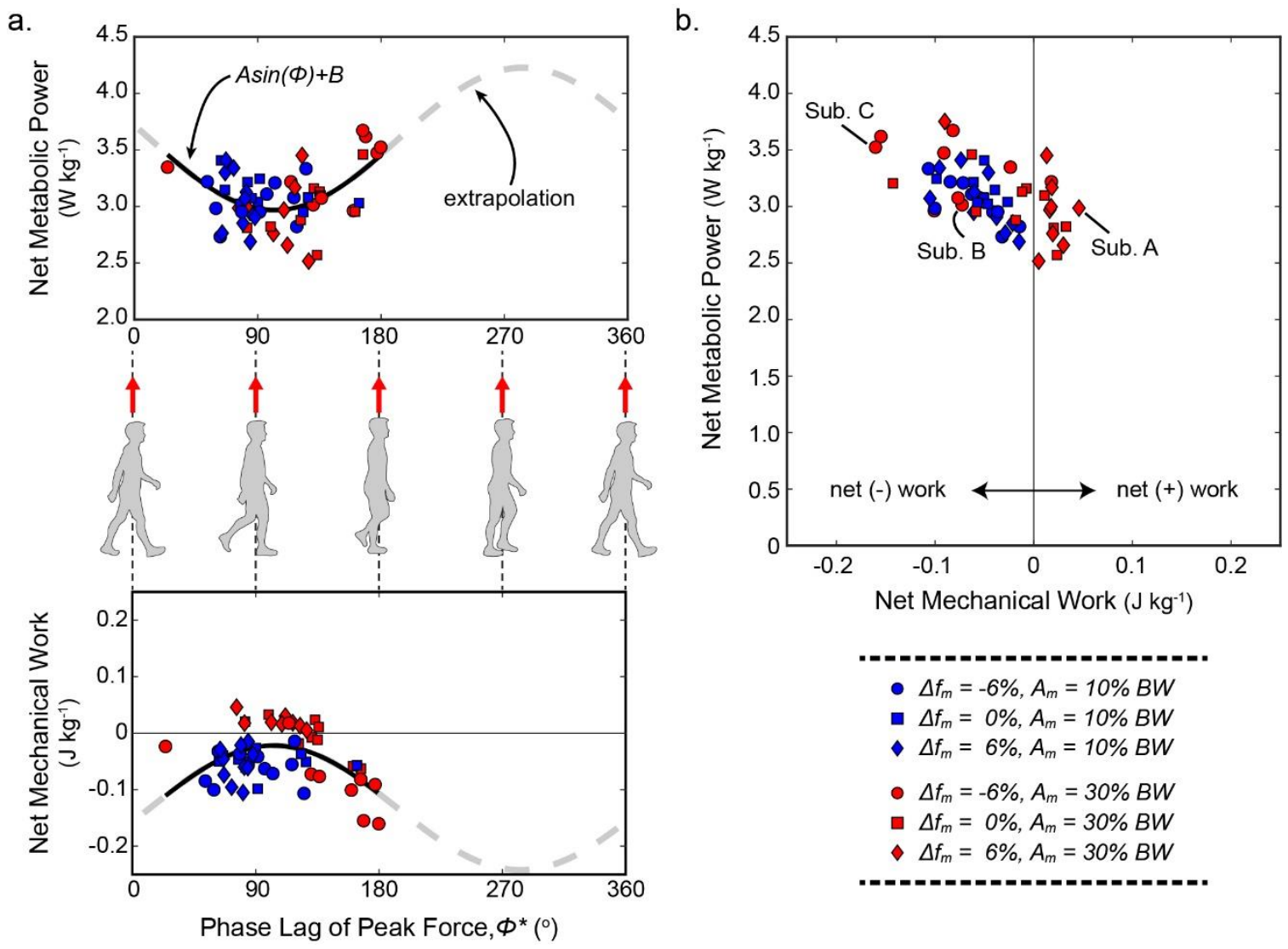

Figure 8. Positive mechanical work from the oscillator decreases metabolic power. Average data are shown for every subject in each trial condition during the first five minutes of oscillations when no metronome is present. (a) Net metabolic power is plotted versus the phase lag of peak upward force from the oscillations. A phase lag of $0^{\circ}$ (or $\left.360^{\circ}\right)$ corresponds to peak upward force occurring at double stance while a lag of $180^{\circ}$ corresponds to peak upward force occurring at midstance (i.e. when the center of mass passes over the stance foot). Net mechanical work done on subjects by the oscillation forces is also plotted versus the phase lag of peak upward force. Arbitrary sinusoidal functions are fit to these data to indicate the cyclical relationship between center of mass motion and oscillation forces. (b) Net metabolic power decreases as a function of net mechanical work done on subjects by the oscillation forces. A

795 linear mixed model was used to assess the effect of net work on metabolic power (both 796 variables non-dimensionalized; see results in Table S1 in the Supplementary Materials). Example 797 data points from subjects A, B and C are labelled for comparison in plots of Figure 7. Blue and 798 red data points correspond to trial conditions where $A_{m}=10,30 \% B W$ respectively. Circles, 799 squares and diamonds correspond to trial conditions where $\Delta f_{m}=-6,0,6 \%$ respectively. 

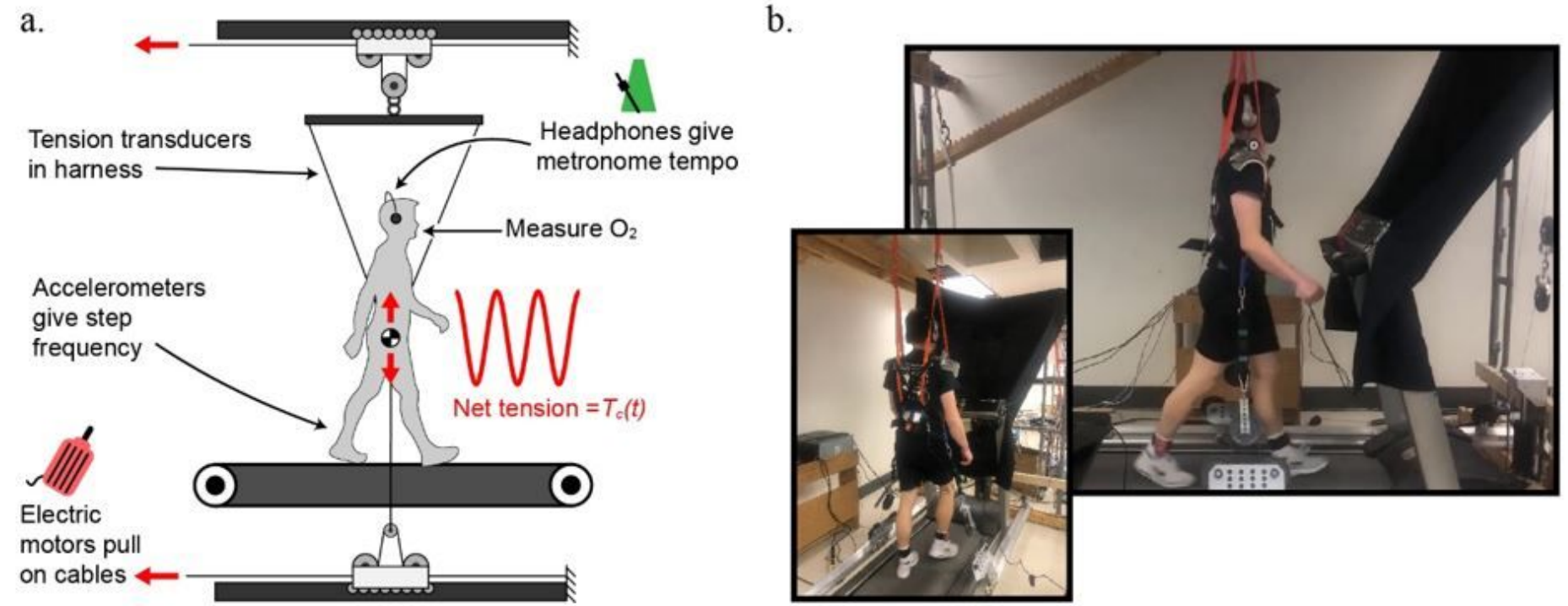

\section{Figure 1}

System schematic and images. (a) A schematic of the oscillator system is depicted in the sagittal plane. (b) Images of a subject walking in the system during a trial, from the side and from behind. Downward force came as the resultant of self-equalizing oblique cables. A curtain was used to blind the subject from any motion of the pulleys or motors, and headphones were used to play ambient noise to block out rhythmic sounds from the system. The headphones were also used to play a metronome beep during portions of the experiment. A more detailed description of the system can be found in the Supplementary Materials.

a.

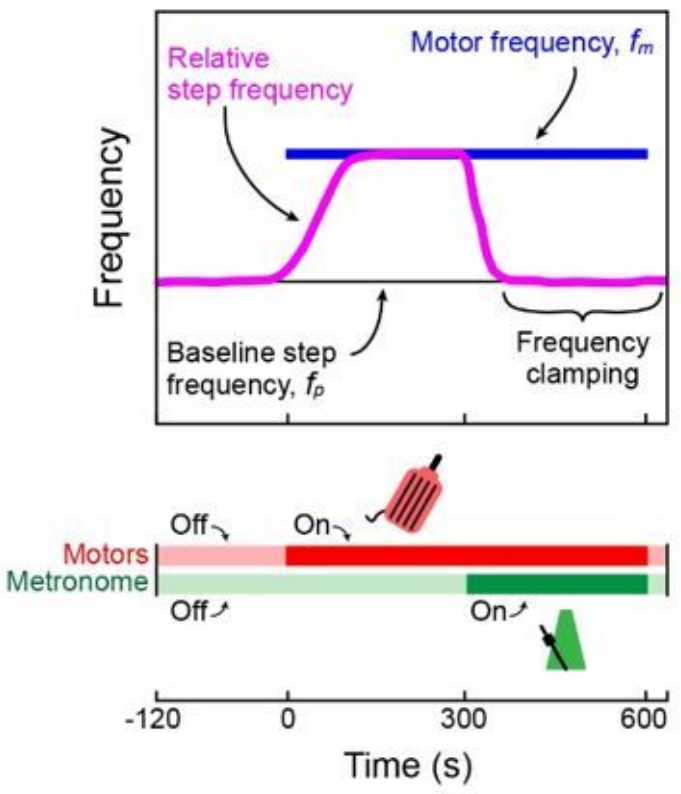

b.
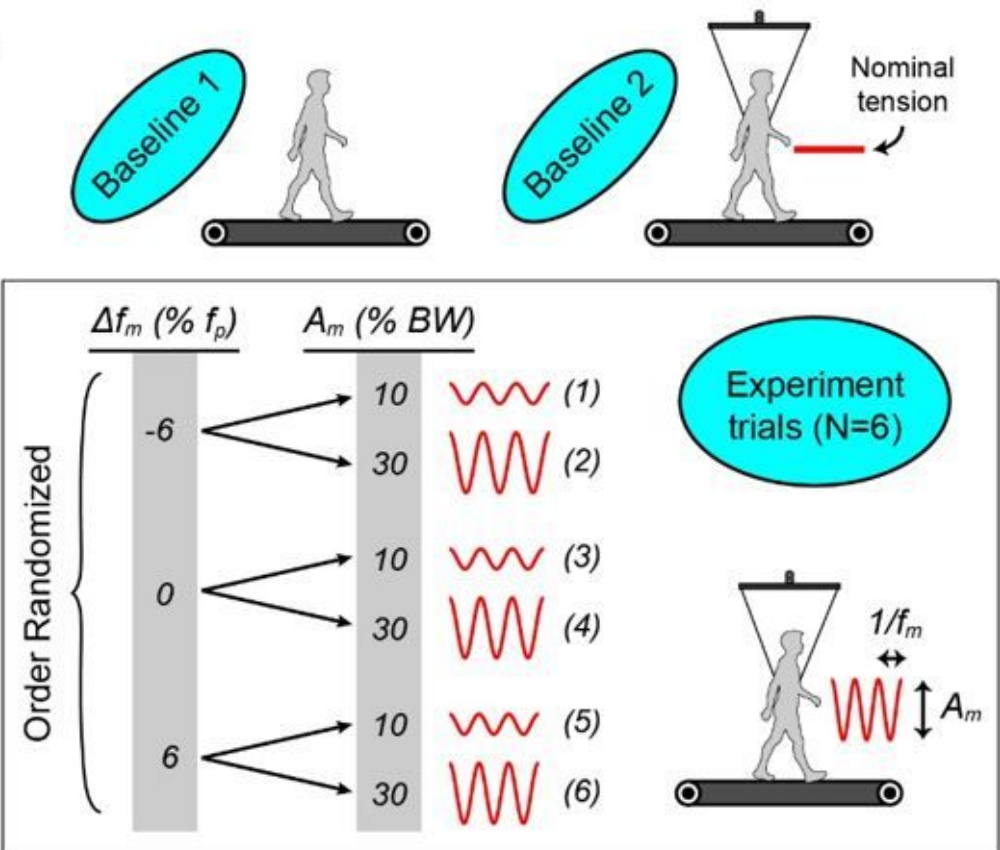
Figure 2

Experimental protocol. (a) A generic trial condition is depicted with simulated step frequency data over time (magenta) and constant motor frequency (blue). Subjects walk with no oscillations during the first two minutes of the test. Motor oscillations begin at time zero and continue for five minutes while subjects freely interact with the system. Oscillations continue for another five minutes, but now a metronome directs individuals to step at their baseline preferred frequency (f_p) despite the external oscillation frequency ("frequency clamping"). The oscillations and metronome are terminated, and the subject is given fifteen additional seconds to prepare for the end of the trial. (b) Baseline conditions and oscillation parameters during experiment trials are shown: $\Delta f \_m=0, \pm 6 \%$ and $A \_m=10,30 \%$ body weight (BW). Trial conditions were implemented randomly to reduce ordering effects.
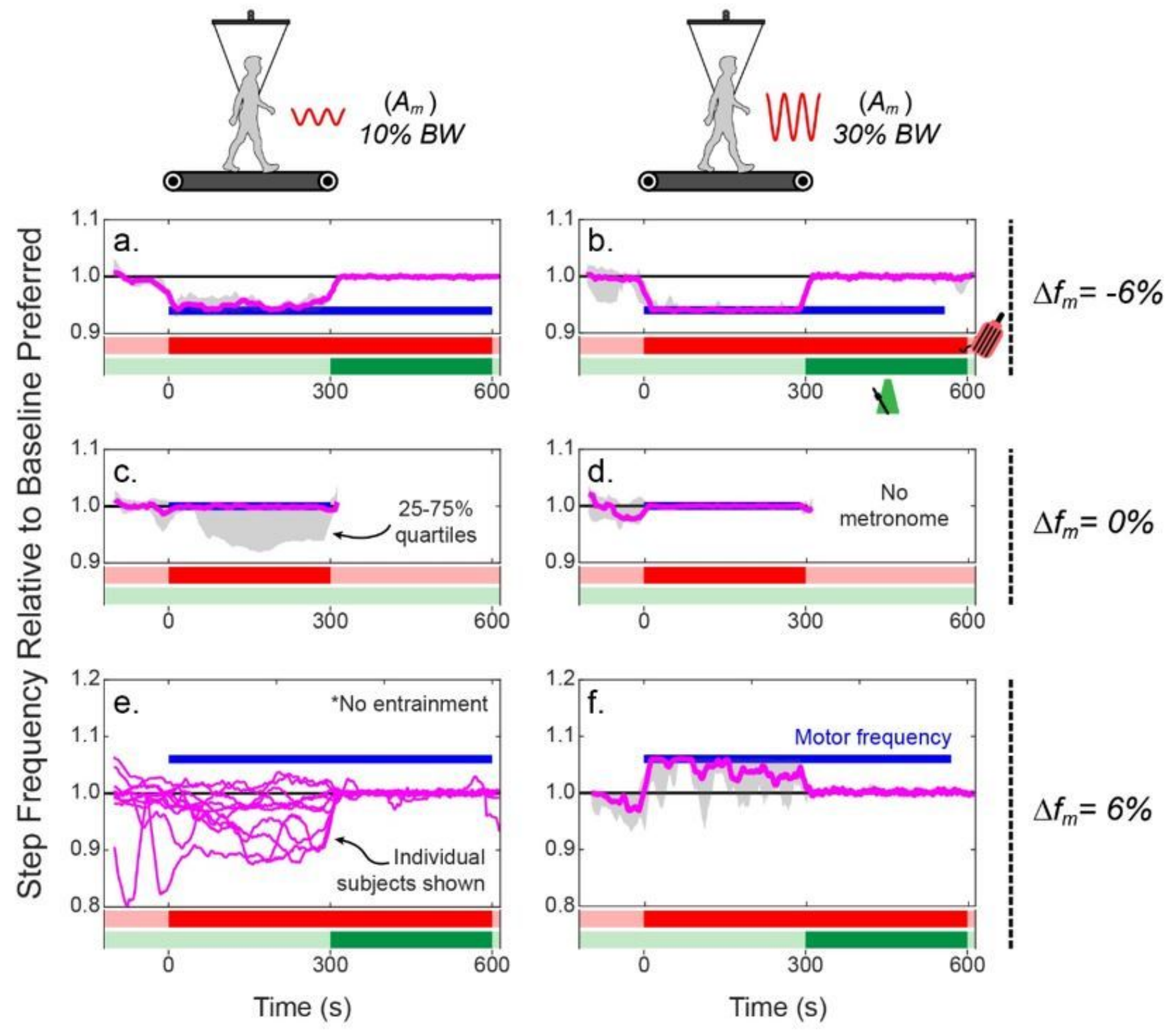

$\Delta f_{m}=0 \%$

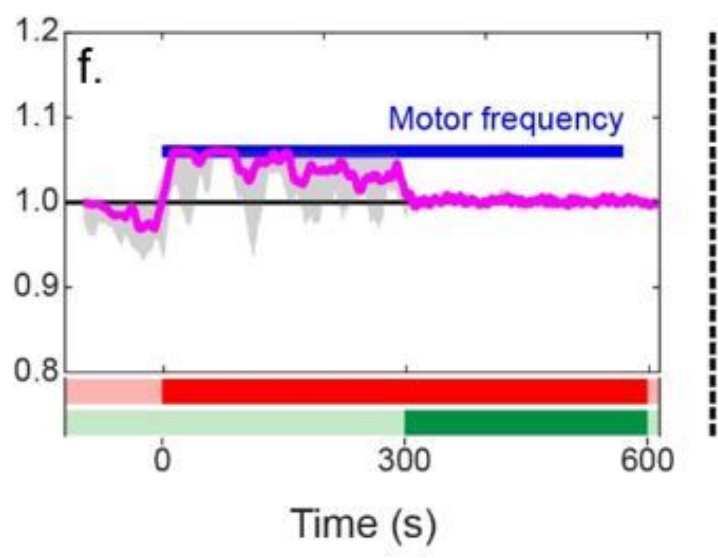

$\Delta f_{m}=6 \%$

Figure 3 
Entrainment results. The median relative step frequency (f_r, step frequency divided by preferred step frequency in Baseline 2; magenta) of all subjects who entrained is plotted over each trial duration. $25 \%$ and $75 \%$ quartiles are used to indicate the distribution at every time point (grey shaded area). All trial conditions are shown, including: $\Delta \mathrm{f} \_\mathrm{m}=-6 \%$ in (a) and (b); $\Delta \mathrm{f} \_\mathrm{m}=0 \%$ in (c) and (d); $\Delta \mathrm{f} \_\mathrm{m}=6 \%$ in (e) and (f); A_m $=10 \%$ BW in (a), (c) and (e); A_m $=30 \%$ BW in (b), (d) and (f). The oscillations began at Time $=0 \mathrm{~s}$ and ended at approximately Time $=600 \mathrm{~s}$. During $0 \leq \mathrm{Time} \leq 300 \mathrm{~s}$, subjects responded freely to the force oscillations. During $300 \leq$ Time $\leq 600 \mathrm{~s}$, subjects were directed to follow the cadence of the metronome at their predetermined baseline step frequency ("frequency clamping") even as the oscillations continued at a different frequency. There was no metronome used in trials where $\Delta f_{-} m=0$, since frequencies were already matched. As a result, these experiments ended after around Time=300 s. Note, median data are only shown for individuals who entrained at least once throughout the trial. In the trial condition where $\Delta \mathrm{f} \_\mathrm{m}=6 \%$ and $\mathrm{A} \_\mathrm{m}=10 \% \mathrm{BW}$, individual subject data are shown instead since no entrainment occurred.
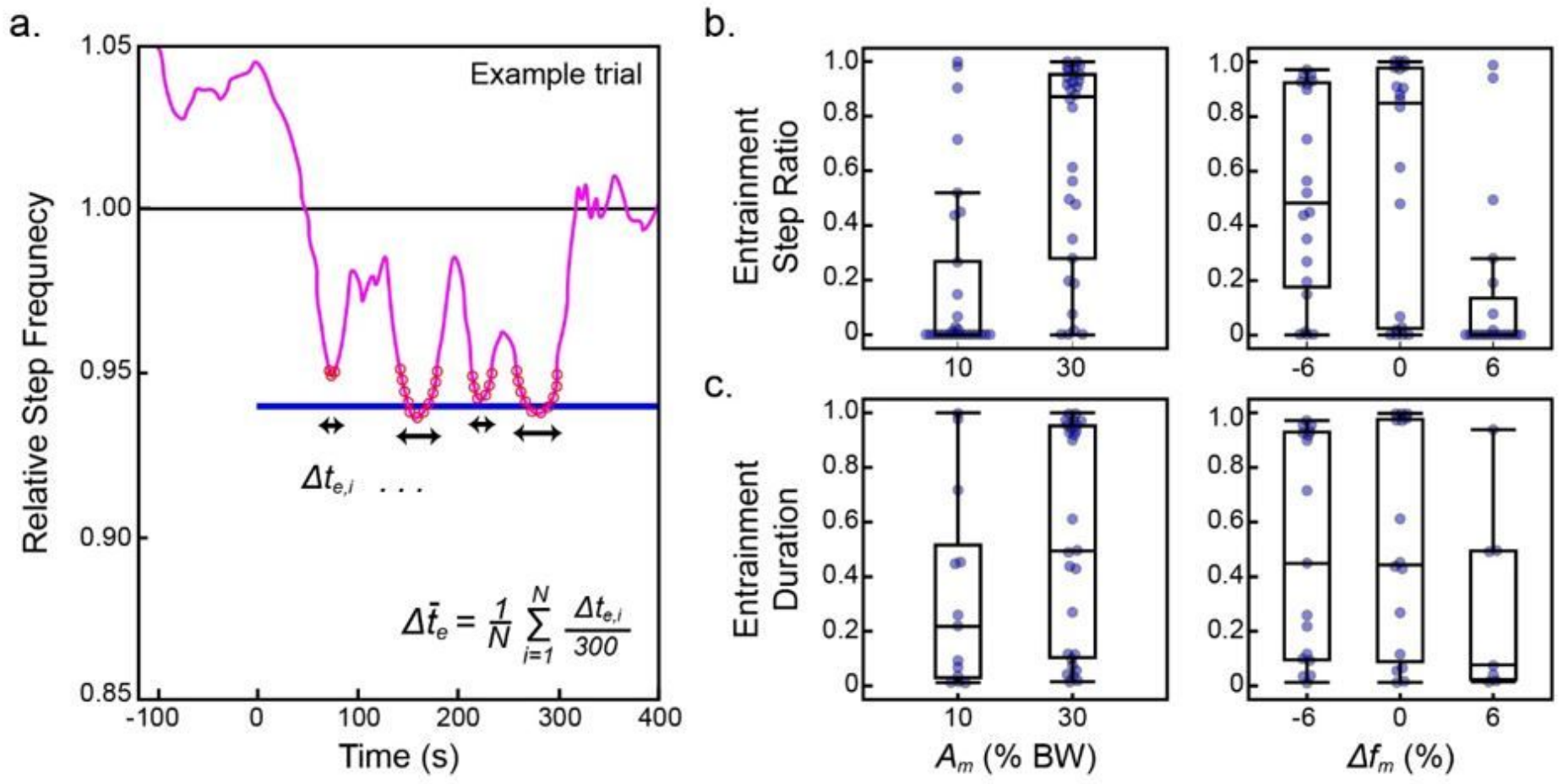

\section{Figure 4}

Entrainment is often transient. (a) Data from an example subject illustrates transient entrainment where relative step frequency (magenta) oscillates towards and away from the motor frequency (blue). Red data points indicate when the subject is considered entrained with the oscillator system (see Methods section for details on entrainment definition). (b) The entrainment step ratio (ESR; ratio of entrained steps to total steps taken during the first five minutes of oscillations in the experiment) and (c) the average entrainment duration ( $\Delta \mathrm{t} \mathbb{}_{\textrm{e}}$; average time duration of bouts of entrainment) are shown as a function of oscillation amplitude and motor frequency, where each data point represents a subject's level of entrainment during each trial and box plots summarize the distribution. Linear mixed models were used to statistically test 
the effects of trial conditions on both entrainment metrics shown here (see Table S1 in the Supplementary Materials for full results).

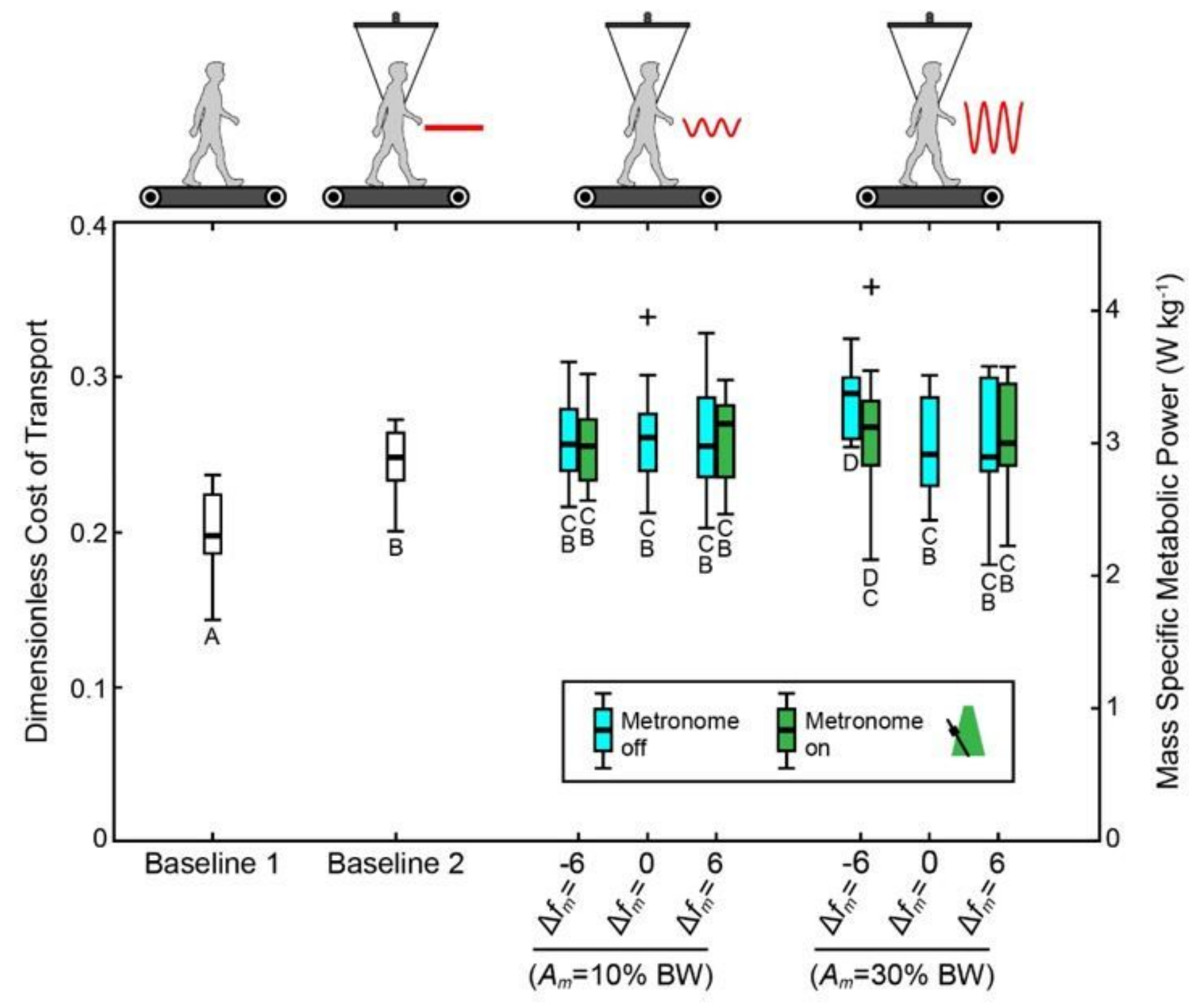

Figure 5

Metabolic power does not depend on entrainment. Non-dimensional metabolic power was compared using a mixed linear regression stratified by trial condition and baseline type. A post hoc Tukey Honestly Significant Difference test was used to compare estimates of metabolic power in the model. Box plots are labelled with letters indicating conditions where power is not significantly different. Outliers are marked with " + ". See Table S2 in the Supplementary Materials for full results. Baseline 1 and 2 refer to walking on the treadmill without and with the harness, respectively. Box plots are shown for all metabolic data collected during the first five minutes of oscillations where the subject freely responded to the oscillation forces (i.e. metronome off). During the next five minutes of oscillations, a metronome guided subjects to 
step at their baseline preferred frequency (importantly, not matched to the oscillation frequency). This allowed for metabolic cost comparisons between entrained and non-entrained gait.

Baseline Interaction
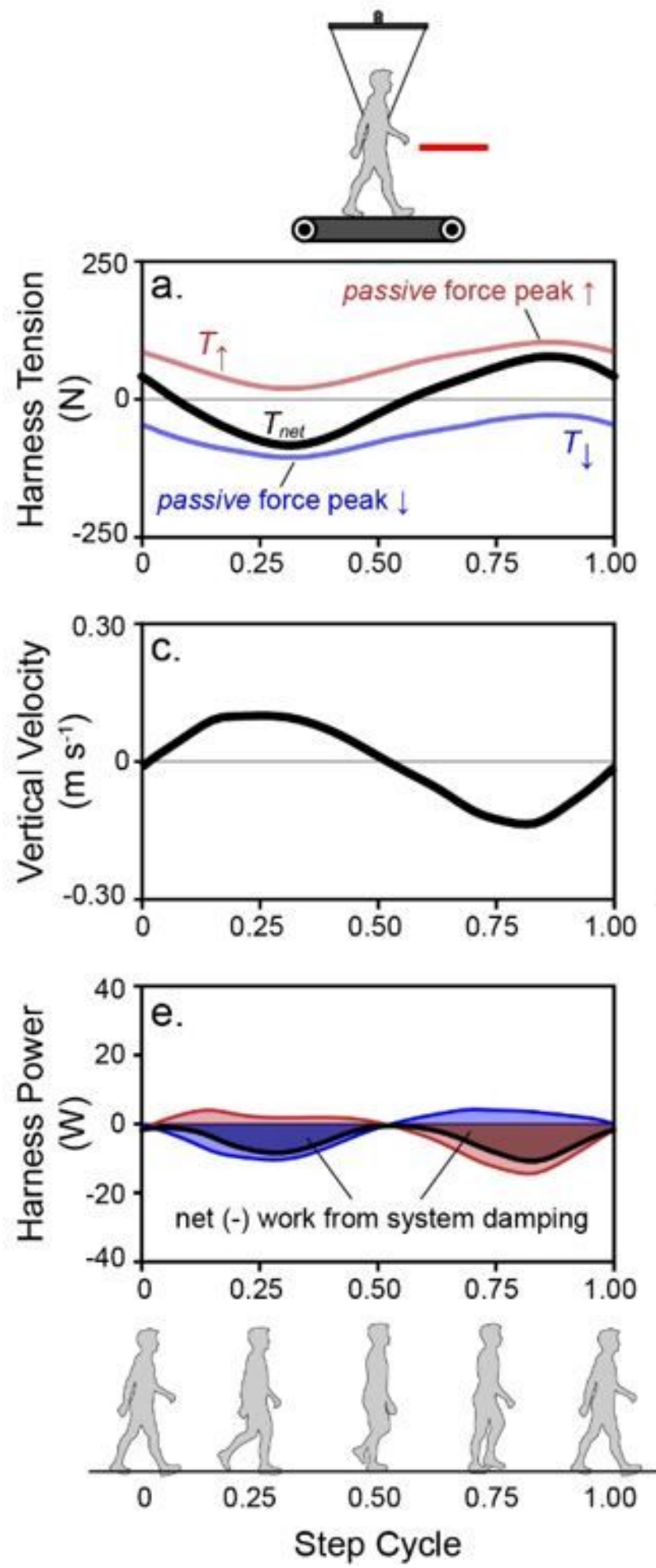

Entrained Interaction
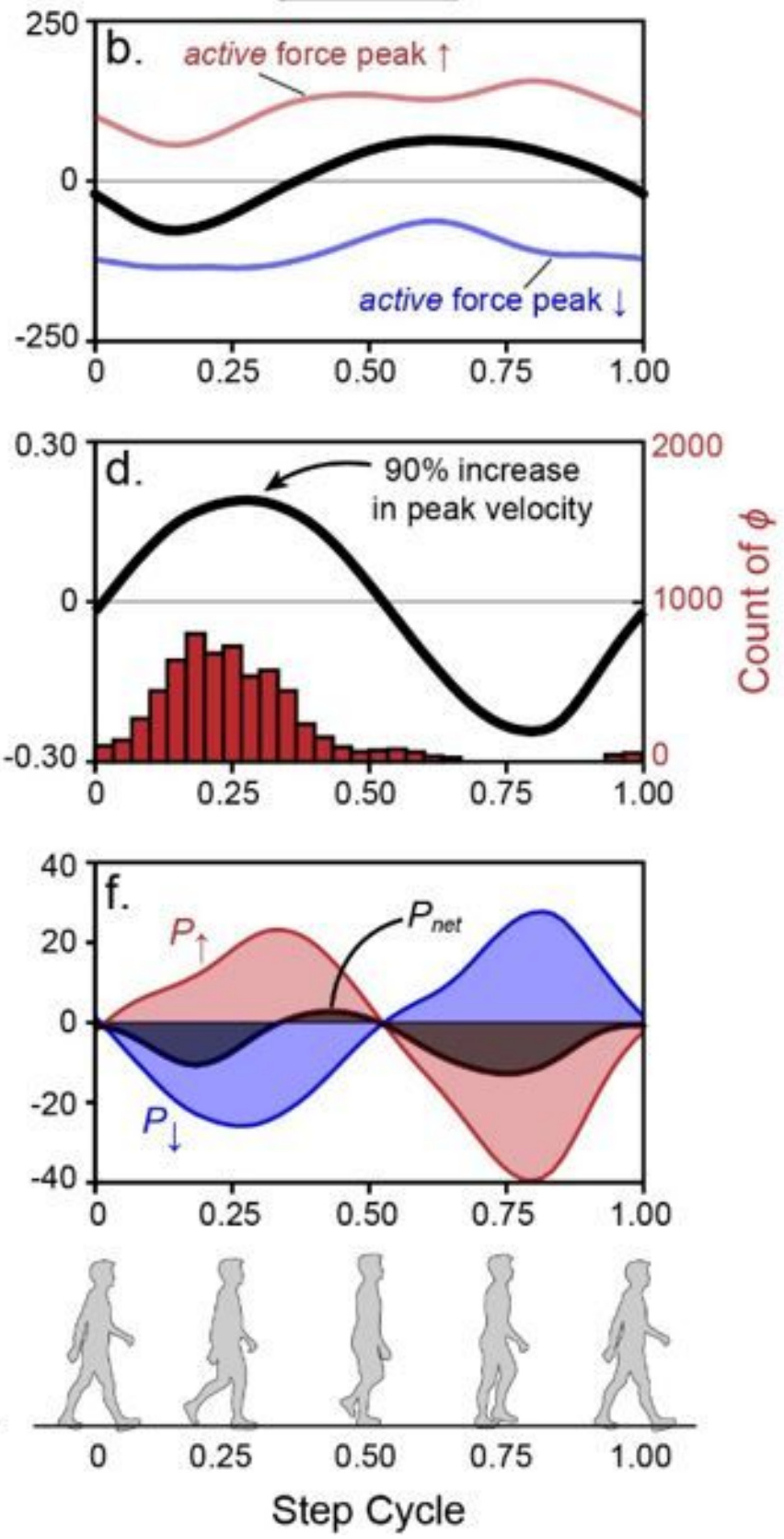

Figure 6

Subjects prefer to align peak oscillation forces at toe off. The average harness tension is shown in (a) and (b), where the red and blue curves indicate cable tension pulling up (T_囚) and down (T_囚), respectively, and black indicates net tension (T_net). Average center of mass vertical velocity is shown in 
(c) and (d), while the red histogram in (d) indicates the distribution of motor phase chosen by subjects

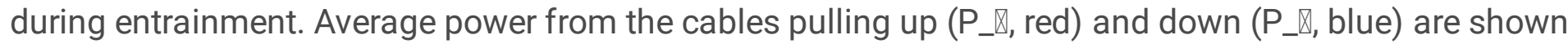
in (e) and (f), while net power (P_net) is indicated with black shading. Panels (a), (c) and (e) indicate average curves measured during Baseline 2 (walking with the harness but no active oscillations), while panels (b), (d) and (f) indicate average curves measured during experiment trials. Snapshots of the walking step cycle are shown near the bottom to help orient the reader to the timing of events shown in plots. Zero in the step cycle corresponds with double stance while 0.5 corresponds with single stance.
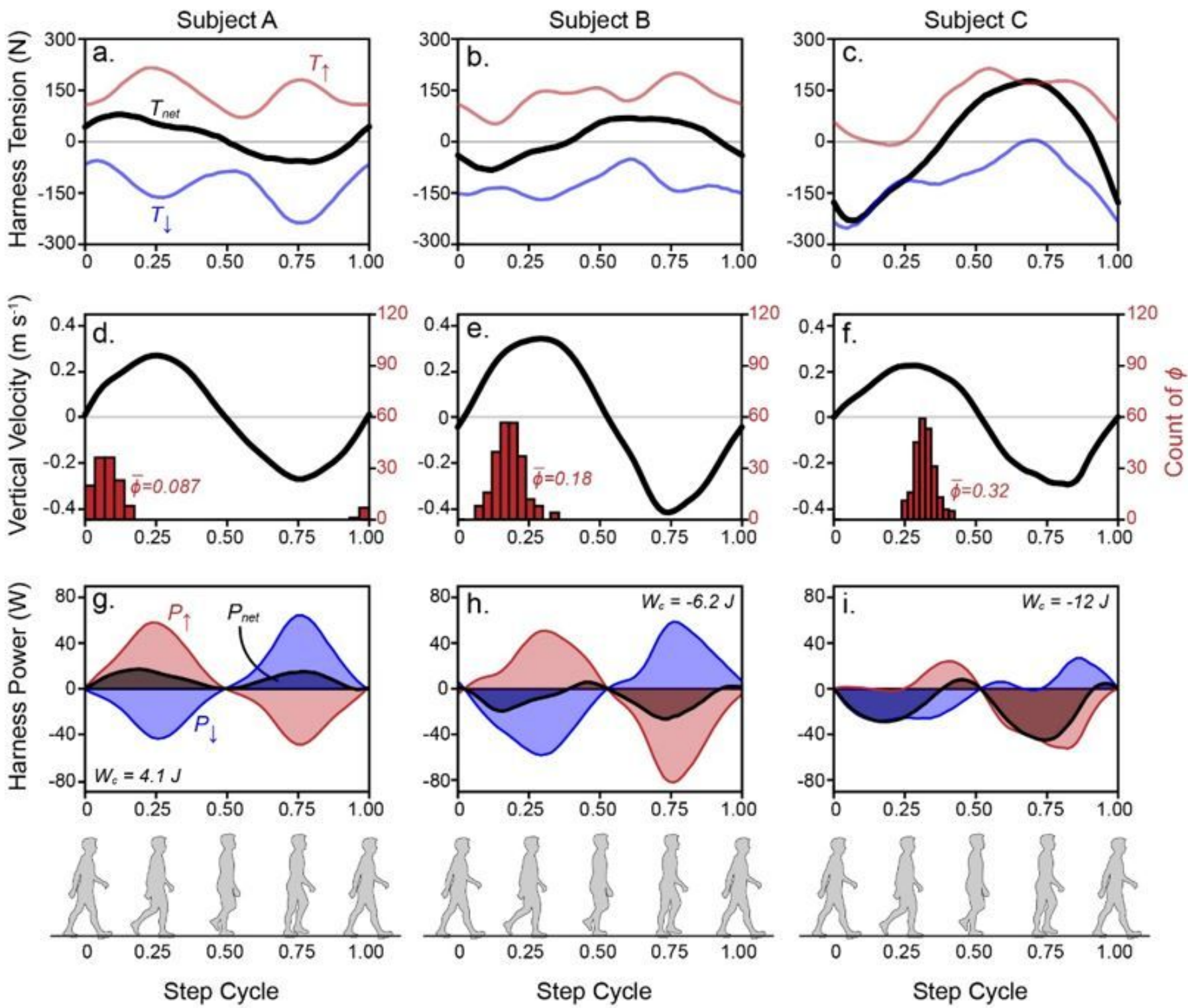

\section{Figure 7}

Subjects prefer a range of phase alignments, resulting in variable net power. Data from three subjects were chosen to demonstrate varying entrainment strategies. Average data for Subject $A\left(\Delta f_{-} m=6 \%\right.$, A_m $=30 \%$ BW, 297 steps averaged) are shown in (a), (d) and (g), where motor phase alignment occurs at $\Delta \nabla=0.087$ of the gait cycle. This strategy aligns peak tension forces with the center of mass (CoM) 
vertical velocity and thus, results in net positive work $\left(W_{-} \mathrm{C}=4.1 \mathrm{~J}\right)$. Average data for Subject $B\left(\Delta \mathrm{f}_{-} \mathrm{m}=-6 \%\right.$, A_m $=30 \%$ BW, 475 steps averaged) are shown in (b), (e) and (h) where motor phase alignment occurs at $\mathbb{Q}$ $\bigotimes=0.18$ of the gait cycle. Since peaks in tension due to motor forces occur slightly later, positive power is relatively low and net negative work occurs $\left(W_{-} \mathrm{C}=-6.2 \mathrm{~J}\right)$. Average data for Subject $\mathrm{C}\left(\Delta \mathrm{f}_{-} \mathrm{m}=-6 \%\right.$, A_m $=30 \%$ BW, 444 steps averaged) are in (c), (f) and (i) where $\mathbb{\nabla}=0.32$ of the gait cycle, and mechanical power from the harness tension is dominated by resistive inertial forces, thus leading to substantial net negative work on the CoM (W_C=-12 J). Data points for subjects $A, B$ and $C$ are labelled in Figure $8 \mathrm{~b}$.
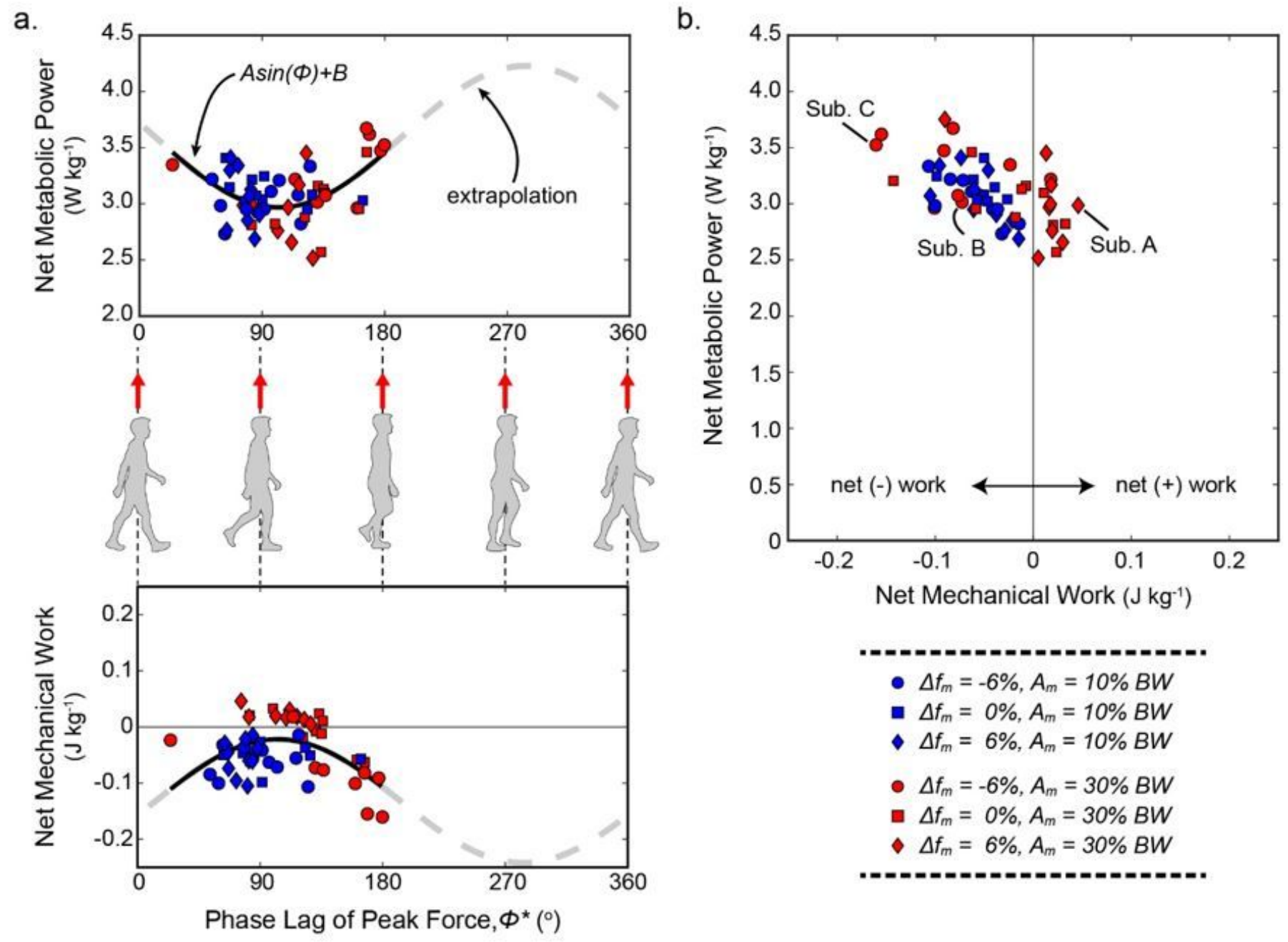

\section{Figure 8}

Positive mechanical work from the oscillator decreases metabolic power. Average data are shown for every subject in each trial condition during the first five minutes of oscillations when no metronome is present. (a) Net metabolic power is plotted versus the phase lag of peak upward force from the oscillations. A phase lag of $0^{\wedge} \mathrm{O}\left(\right.$ or $\varangle 360 \mathbb{\Xi}^{\wedge} \mathrm{O}$ ) corresponds to peak upward force occurring at double stance while a lag of $₫ 180 \rrbracket^{\wedge} \mathrm{O}$ corresponds to peak upward force occurring at midstance (i.e. when the center of mass passes over the stance foot). Net mechanical work done on subjects by the oscillation forces is also plotted versus the phase lag of peak upward force. Arbitrary sinusoidal functions are fit to these data 
to indicate the cyclical relationship between center of mass motion and oscillation forces. (b) Net metabolic power decreases as a function of net mechanical work done on subjects by the oscillation forces. A linear mixed model was used to assess the effect of net work on metabolic power (both variables non-dimensionalized; see results in Table $\mathrm{S} 1$ in the Supplementary Materials). Example data points from subjects $A, B$ and $C$ are labelled for comparison in plots of Figure 7. Blue and red data points correspond to trial conditions where $A \_m=10,30 \%$ BW respectively. Circles, squares and diamonds correspond to trial conditions where $\Delta \mathrm{f} \_\mathrm{m}=-6,0,6 \%$ respectively.

\section{Supplementary Files}

This is a list of supplementary files associated with this preprint. Click to download.

- SupplementaryMaterials.pdf 